\title{
On 3-Harness Weaving: Cataloging Designs Generated by Fundamental Blocks Having Distinct Rows and Columns
}

\author{
Shelley L. Rasmussen \\ Department of Mathematical Sciences \\ University of Massachusetts, Lowell, MA, USA \\ Submitted: May 1, 2007; Accepted: Dec 5, 2007; Published: Jan 1, 2008 \\ Mathematics Subject Classification: 05B45
}

\begin{abstract}
A weaving drawdown is a rectangular grid of black and white squares with at least one black and one white square in each row and column. A pattern results from vertical and horizontal translations of the defining grid. Any such grid defines a tiling pattern. However, from a weaving point of view, some of these grids define actual fabrics while others correspond to collections of threads that fall apart. This article addresses that issue, along with a discussion of binary representations of fabric structures. The article also catalogs all weaving (or tiling) patterns defined by grids having three distinct columns and three to six distinct rows, and groups these patterns into design families based on weaving symmetries.
\end{abstract}

\section{Introduction.}

Weaving is a process of creating a fabric by interlacing a set of yarn strands called the weft with another set of strands called the warp. The lengths of yarn called warp ends are tied in parallel and held under tension on the weaving device or loom. At each step in the weaving process, the weaver separates warp ends into two layers, upper and lower, passes a weft strand through the resulting opening (called the shed), then moves or beats that weft strand so that it lies against previously woven weft yarns, perpendicular to the warp. Lifting another subset of warp ends, the weaver repeats the process until the fabric is completed. 


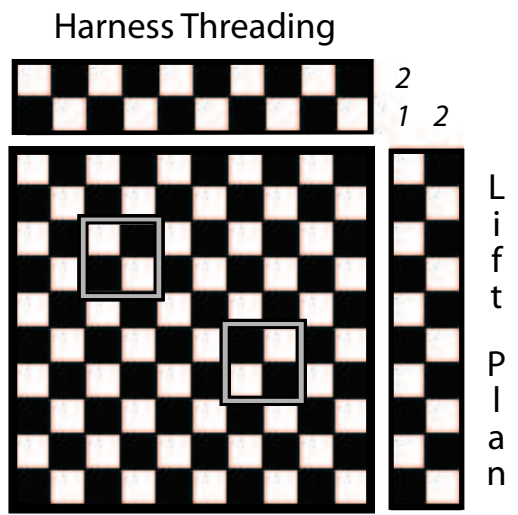

(a) plain weave

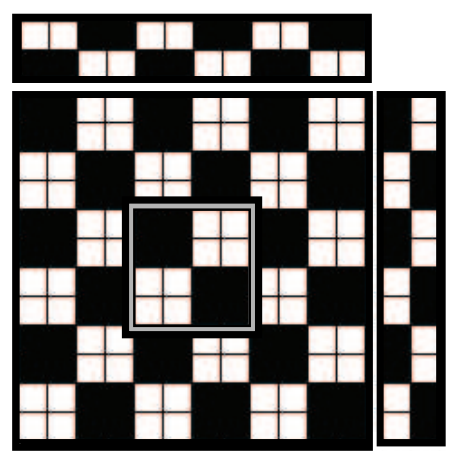

(b) basket weave

Figure 1: (a) A weaver's draft of plain weave fabric structure. Each of the two outlined $2 \times 2$ blocks is sufficient to define plain weave. (b) Draft of a basket weave defined by the outlined $4 \times 4$ block.

A loom with a harness mechanism aids the weaving process. If a warp thread is attached to a harness, the thread rises and falls with that harness. The simplest such loom has two harnesses, sufficient to create the fabric structure called plain weave or tabby. With even-numbered warp ends passed through one harness and oddnumbered through the other, the weaver lifts the harnesses alternately to produce the familiar checkerboard look of plain weave illustrated in Figure 1a.

The weaver's draft in Figure 1a shows ten warp and ten weft threads, although two of each would be sufficient to define the plain weave structure. Following textile industry practice, warp ends are shown here in black and weft in white [25]. A black square indicates that a warp end is lifted and therefore passes over the weft yarn, while a white square indicates weft passing over warp. The $2 \times 10$ rectangle at the top of the draft is the threading diagram, with harnesses numbered from bottom to top, showing how warp yarns pass through the harnesses. Numbering warp ends from left to right, the first row of the threading diagram shows that the odd-numbered warp threads pass through harness 1 , evens through harness 2 . The $10 \times 2$ rectangle at the right of the draft shows the harness lifting plan. With harnesses numbered from left to right, column 1 contains a black square when harness 1 is lifted, column 2 is black when harness 2 is lifted. To produce the exact pattern shown in Figure 1a, the weaver starts at the bottom of the draft and passes the first weft thread through the shed with harness 1 (odd-numbered warp ends) lifted, passes the second weft 
through with harness 2 (even-numbered warp ends) lifted, and so on, creating the $10 \times 10$ grid of fabric represented in the bottom left of the diagram. This $10 \times 10$ grid, called the drawdown, defines the fabric.

In any drawdown, each row and column must contain at least one white and one black square [18]. Grübaum and Shephard [7] pointed out that this requirement is not sufficient to guarantee that a draft represents a weaving that "hangs together". A number of authors have addressed this issue, including Lourie [18], Clapham [4], Enns [6], Grünbaum and Shephard [8] and Delaney [5], and we will as well.

A drawdown represents the physical interlacement structure of warp and weft. We will focus on this interlacement structure, ignoring for now the design possibilities that come with the use of color.

Using the terminology of Grünbaum and Shephard [7], we say that plain weave is a periodic design or pattern defined by vertical and horizontal translations of either of the $2 \times 2$ fundamental blocks outlined in Figure 1a. From a weaving or tiling point of view, these two blocks are equivalent, since both define the same design when extended over the plane. In general, an $m \times n$ grid of black and white squares is a fundamental block of a pattern if each row and column contains at least one white and one black square and the pattern results from vertical and horizontal translations of this block.

By the above definition, the $10 \times 10$ grid in Figure 1a is a fundamental block representing the plain weave fabric structure, as are the $2 \times 10$ and $10 \times 2$ rectangles in that figure. However, the $2 \times 2$ fundamental blocks are the smallest blocks we can use to define plain weave and are therefore irreducible or basic blocks. In general, we will say a fundamental block is a basic block if it is irreducible in the sense that no block with fewer rows or columns defines the same pattern.

Many patterns are generated by basic blocks that have some identical rows and/or columns. One such pattern is the basket weave illustrated by the draft in Figure 1b. This basket weave is a variation on plain weave in that it can be woven on two harnesses, and we call it a 2-harness design, even though the structure is defined by a $4 \times 4$ basic block. In general, we will call a fabric structure a $k$-harness design if $k$ is the minimum number of harnesses required to weave it. A basic block generating a $k$-harness design has exactly $k$ distinct columns [18]. The plain weave in Figure 1a and the basketweave in $1 \mathrm{~b}$ are 2-harness designs, generated by basic blocks having two distinct columns.

We might reasonably ask: How many fabric structures can be woven on a given number of harnesses? Equivalently, how many rectangular-grid two-color tiling patterns result from basic blocks with a given number of distinct columns? Steggall [22] found the number of basic blocks of size $n \times n$ that have exactly one black square in 
each row and column. Grünbaum and Shephard [7], [8], [9], [10] considered classes of patterns they called isonemal fabrics, including satins and twills. Related work on twills and twillins was reported by J.A. Hoskins, W.D. Hoskins, Praeger, Stanton, Street and Thomas (see, for example: [12], [13], [14]).

With the restriction that adjacent rows and columns are not equal, the checkerboard pattern of simple plain weave shown in Figure 1a is the only 2-harness design. How about 3-harness designs? Weaving with three harnesses (or shafts) has a long tradition, as suggested by de Ruiter's [21] discussion of three-harness designs and an analysis of 18th and 19th century textiles by Thompson, Grant and Keyser [24]. However, this author has not found a study of the number of patterns that can be woven on three harnesses. In later sections, we will begin this study by finding the number of patterns generated by $m \times 3$ basic blocks having no equal rows or columns. We will also group these patterns into families or equivalence classes of fabric designs based on weaving symmetries and illustrate these design families.

Before proceeding, however, we must address the problem of determining whether or not a weaving hangs together. Such a determination is easier if we represent drafts with binary matrices, as discussed in the next section.

\section{$2 \quad$ Weaving and binary matrices}

We can display the interlacement structure of a fabric consisting of $m$ weft and $n$ warp threads as an $m \times n$ grid of black and white squares, called the drawdown. An alternative representation of the fabric structure is an $m \times n$ matrix of 0's and 1's, with 1 indicating a warp thread passing over weft (black square in the drawdown) and 0 otherwise. We will refer to this binary representation as the drawdown matrix.

Lourie [18] and Hoskins [11], among others, discussed the idea of factoring an $m \times n$ drawdown matrix into a product of two matrices, one representing the warp threading and the other, the lift plan. Let $D$ denote the $m \times n$ drawdown matrix of an $h$-harness design (that is, there are $h$ distinct columns in $D$ ). Using the notation of Lourie [18], define the harness threading matrix $H$ as the $h \times n(0,1)$-matrix with rows 1 through $h$ representing harnesses 1 through $h$, respectively, and columns corresponding to warp threads numbered from left to right. $H$ has a 1 in position $(i, j)$ if warp thread $j$ passes through harness $i$, and 0 otherwise. (This mathematical definition of $H$ reverses the row order traditionally used by weavers at the top of a draft to illustrate harness threading.) Define the lift plan matrix $L$ as the $m \times h$ matrix that has a 1 in position $(i, j)$ if all of the warp threads lifted by harness $j$ pass over the weft thread corresponding to row i of the drawdown, 0 otherwise. Then, $L \times H=D$. 
Consider, for example, the basketweave defined by $4 \times 4$ basic block $b$ outlined in Figure 1b. If $D$ represents the drawdown matrix corresponding to $b$, the matrix equation $L \times H=D$ becomes:

$$
\left(\begin{array}{ll}
1 & 0 \\
1 & 0 \\
0 & 1 \\
0 & 1
\end{array}\right) \times\left(\begin{array}{llll}
1 & 1 & 0 & 0 \\
0 & 0 & 1 & 1
\end{array}\right)=\left(\begin{array}{llll}
1 & 1 & 0 & 0 \\
1 & 1 & 0 & 0 \\
0 & 0 & 1 & 1 \\
0 & 0 & 1 & 1
\end{array}\right)
$$

In this case, the lift plan matrix $L$ is the transpose of the harness threading matrix $H$, and the resulting drawdown matrix $D$ is symmetric.

Suppose a pattern is generated by an $m \times n$ basic block $b$ whose $n$ columns are all distinct. In such a case, the block itself gives all the information necessary for threading the loom and weaving; we do not require the harness threading and lift plan portions of the weaver's draft. We state this in the following theorem:

Theorem 1. Suppose $b$ is an $m \times n$ basic block whose $n$ columns are all distinct. If $D$ is the drawdown matrix for $b$, then we can write $D=L \times H$, where the harness threading matrix $H$ equals the $n \times n$ identity matrix and the lift plan matrix $L$ equals $D$.

Proof. For threading such a design, we can use what weavers call a straight draw [2]: warp thread $\mathrm{j}$ passes through harness $\mathrm{j}$ for $\mathrm{j}=1, \ldots, n$. Then the threading matrix $H$ is the $n \times n$ identity matrix $I$, and $D=L \times H=L \times I=L$.

For example, consider the $4 \times 4$ block $b$ outlined in Figure 2a. Both $b$ and its drawdown matrix $D$ have four distinct columns and the matrix equation $L \times H=D$ is:

$$
\left(\begin{array}{llll}
0 & 0 & 1 & 1 \\
0 & 0 & 1 & 0 \\
1 & 1 & 0 & 0 \\
1 & 0 & 0 & 0
\end{array}\right) \times\left(\begin{array}{llll}
1 & 0 & 0 & 0 \\
0 & 1 & 0 & 0 \\
0 & 0 & 1 & 0 \\
0 & 0 & 0 & 1
\end{array}\right)=\left(\begin{array}{llll}
0 & 0 & 1 & 1 \\
0 & 0 & 1 & 0 \\
1 & 1 & 0 & 0 \\
1 & 0 & 0 & 0
\end{array}\right)
$$

In Sections 4 through 7, we consider only basic blocks whose columns are all distinct. All patterns are generated by horizontal and vertical translations of the generating block $b$. Then $b$ provides all the information normally provided in a draft, describing the fabric structure, threading and lift plan. 


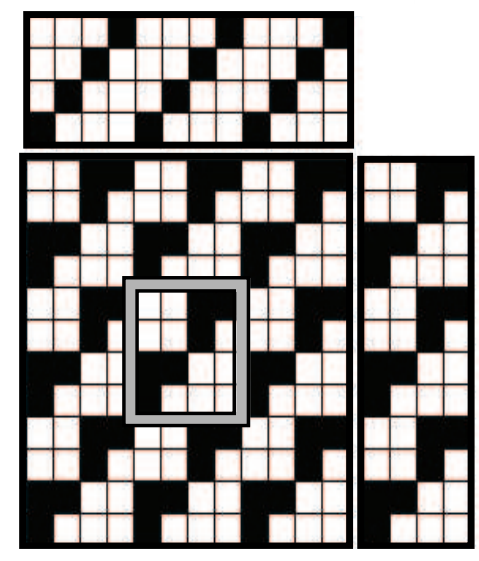

(a)

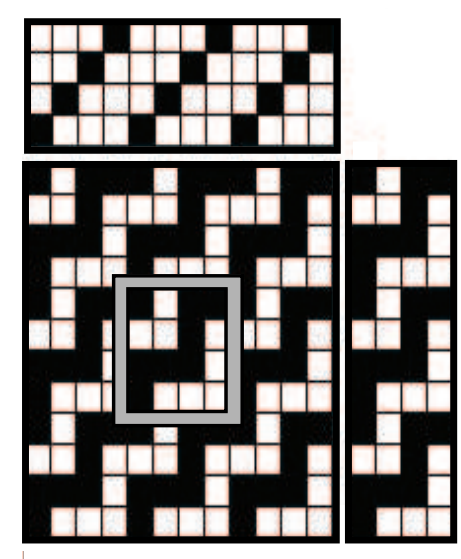

(b)

Figure 2: (a) A weaver's draft of a fabric that hangs together, with its $4 \times 4$ basic block outlined. (b) Draft of a weaving structure that does not hang together. The $4 \times 4$ basic block that defines the tiling pattern is outlined.

\section{When a weaving hangs together}

Consider the tiling patterns in Figure 2. Each is a 4-harness design that can be represented by a $4 \times 4$ basic block having at least one black and one white square in each row and column and each can be used to produce a weaving. Following the draft in Figure 2a results in a fabric with interlacement structure indicated directly by the pattern of black and white squares in the draft. This is not the case for the draft in Figure 2b. Weaving from this draft results in two separate plain weave fabrics: whenever either harness 2 or 4 is lifted, so are harnesses 1 and 3, so that the fabric involving harnesses 2 and 4 lies below that involving harnesses 1 and 3 .

Weavers call the fabric structure in Figure $2 \mathrm{~b}$ doubleweave and use it in a number of ways. If separate weft threads are used for each row of the design, two completely separate plain weave fabrics result: one is woven above the other and the two fabrics can be lifted apart. Handweavers generally wrap a single long length of weft yarn on a shuttle and then pass the shuttle back and forth through the warp. The order in which the harnesses are lifted then determines topological properties of the fabric. If a weaver follows the draft in Figure $2 \mathrm{~b}$ with a single long weft thread that starts from the right side of the loom, as shown in Figure 3a, the two resulting plain weave fabrics are locked together at each side in the form of a flattened cylinder. If the 


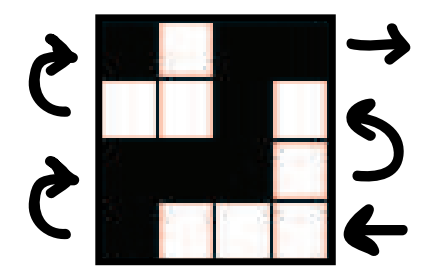

(a)

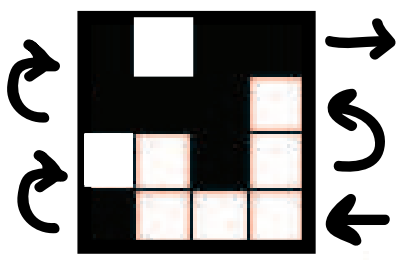

(b)

Figure 3: (a) Weaving with a continuous weft as shown results in two fabric layers locked together on each side, creating a flattened cylinder. (b) Using a continuous weft and weaving as shown results in two fabric layers locked on the right side, opening to a fabric twice as wide as the warp span on the loom.

weaver changes the harness lifting order to that in Figure 3b, the resulting fabric layers are locked only on the right side. When removed from the loom, the weaving can be opened into a single plane of fabric twice as wide as the span of warp threads on the loom, with length corresponding to half the weft passes used in the weaving.

Artists also use doubleweave for decorative purposes. As a simple example, consider the sample of 4-harness doubleweave shown in Figure 4. The weaver used a total of 48 warp threads: 4 dark warp strands in each of harnesses 2 and 4 and 4 light warp strands in each of harnesses 1 and 2 across the middle third of the piece and 24 light warp threads (6 per harness) on each side. The weft is made up of 48 passes with the light-colored yarn. Using harnesses 1 and 3 produces a fabric that is all light-colored; harnesses 2 and 4 result in a fabric with a vertical dark/light checkered stripe down the middle. The weaver wove the bottom and top thirds of the sample with the solid-color fabric layer on top and the middle third with the striped layer on top. The resulting piece, showing 24 warp and 24 weft strands on each side, has the interesting property that two planes of fabric intersect twice. The color pattern in Figure 4 cannot be woven as a single layer. Delaney [5] called such a design essentially reducible.

We can conceive weavings with more than two layers of fabric, although a weaver might find them technically difficult to construct. Albers [1], who dedicated her book to the weavers of ancient Peru, reported that the Peruvians made use of double, triple and quadruple weaves.

For the remainder of this section, we assume that a pattern is generated by an 


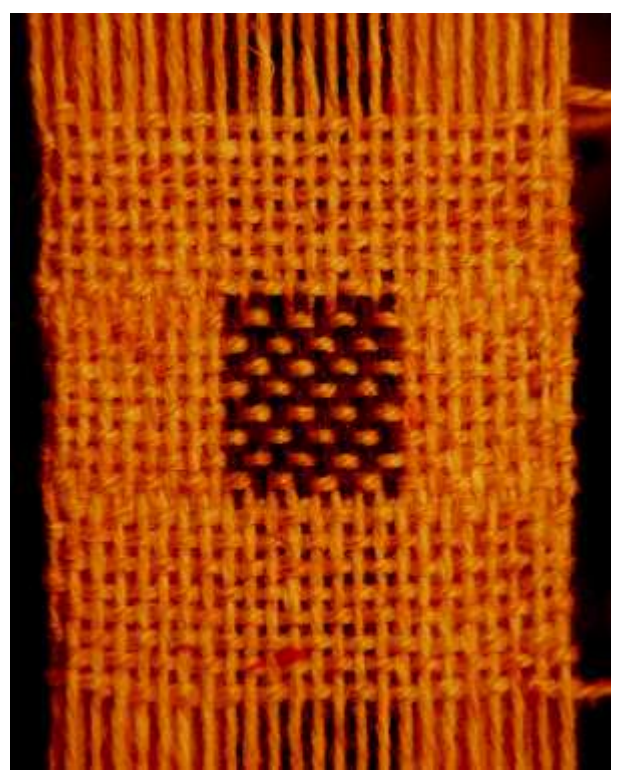

Figure 4: Doubleweave sample with a solid color plain weave fabric twice intersecting a striped plain weave fabric.

$m \times n$ fundamental block. We also assume that the weaver uses individual weft threads to produce the "weaving" from the draft. Then, a weaving "falls apart" if there are sets of threads that can be physically separated. We'll say such sets are mutually unconnected. If a set of threads cannot be pulled apart in this way, we'll say these threads are mutually connected and the corresponding weaving "hangs together".

How can we tell whether or not a drawdown represents a weaving that hangs together? Clapham [4] provided a procedure for such a determination, which we will repeat here. Let rsum $_{i}$ denote the row sum of row $i$ of the drawdown matrix and csum $_{j}$ the column sum of column $j$. Suppose that the rows and columns of the matrix are arranged so that ssum $_{1} \leq$ rsum $_{2} \leq \ldots \leq$ rsum $_{m}$ and csum $_{1} \geq$ ssum $_{2} \geq$ $\ldots \geq \operatorname{csum}_{n}$ (whether a weaving hangs together does not depend on the order of the rows or columns of the drawdown). Let $s$ and $t$ be integers such that $0 \leq s \leq m$ and $0 \leq t \leq n$, excluding the possibility that $(s, t)$ is either $(0,0)$ or $(m, n)$, and define the function $E(s, t)$ this way:

$$
E(s, t)=t(m-s)-\left(\operatorname{csum}_{1}+\ldots+\text { csum }_{t}\right)+\left(\text { rsum }_{1}+\ldots+\text { rsum }_{s}\right)
$$


Clapham [4] proved that $E(s, t) \geq 0$ and that the weaving falls apart if and only if $E(s, t)=0$ for some $(s, t)$, providing a simple method of determining whether a weaving hangs together, repeated below:

\section{Determining Whether a Weaving Hangs Together (Clapham)} If sum $_{1}=0$, take $s=1$ and $t=0$ and the weft strand corresponding to rsum $_{1}$ can be lifted off. If not, for each $t=1, \ldots, n$, find the largest $s$ such that $r$ sum $_{s}<t$ (the row sums are increasing) and evaluate $E(s, t)$ defined above. If any of these equals 0 (excluding $E(m, n)$ ) then the weft strands corresponding to rows with row sums rsum $_{1}, \ldots$, rsum $_{s}$ and the warp threads corresponding to columns with column sums csum $_{1}, \ldots$, csum $_{t}$ can be lifted off. Otherwise the fabric hangs together.

Consider binary matrix representations $D_{a}$ and $D_{b}$ of the basic blocks of Figures $2 \mathrm{a}$ and $2 \mathrm{~b}$, respectively, each rearranged so that row sums are increasing and column sums are decreasing:

$$
D_{a}=\left(\begin{array}{cccc}
1 & 0 & 0 & 0 \\
0 & 1 & 0 & 0 \\
1 & 0 & 1 & 0 \\
0 & 1 & 0 & 1
\end{array}\right) \quad D_{b}=\left(\begin{array}{cccc}
1 & 0 & 0 & 0 \\
0 & 1 & 0 & 0 \\
1 & 1 & 0 & 1 \\
1 & 1 & 1 & 0
\end{array}\right)
$$

The row sums for the binary matrix $D_{a}$ are $1,1,2$ and 2 , the column sums are $2,2,1$ and 1 , and $E(s, t)$ is always greater than 0 . For the matrix $D_{b}$, the row sums are 1,1,3 and 3, the column sums are 3,3,1 and $1, E(2,2)=0$ and $E(s, t)>0$ for all pairs $(s, t)$ other than $(2,2)$. This agrees with our earlier observation that the draft in Figure $2 \mathrm{~b}$ results in two plain weave fabrics, while the draft in Figure 2a results in a single fabric.

Clapham's procedure applies to any draft that can be represented by an $m \times$ $n$ binary array, no matter how many unconnected layers. If the binary matrix is arranged so that row sums are nondecreasing and column sums are nonincreasing, then $E(s, t)=0$ if and only if the first $s$ "row" or weft strands and the first $t$ "column" or warp strands can be lifted off the others. These $s$ weft and $t$ warp threads may make up a single fabric or it may be possible to partition them into separate fabric layers and/or loose strands.

The drawdown matrix for the sample in Figure 4 meets Clapham's criterion for a weaving that hangs together. However, there are three separate horizontal strips 
of doubleweave in this sample, connected where the fabric layers intersect. The appearance of the $48 \times 48$ grid of black and white squares in the draft does not directly correspond to the physical appearance of the woven piece; the sample has just 24 warp and 24 weft threads showing on each side. Similarly, the appearance of the $12 \times 12$ drawdown in Figure 2b does not correspond to the physical appearance of the resulting doubleweave; each side of the woven sample reveals 6 warp and 6 weft strands. We see that a weaving may "hang together" but not consist of a single fabric layer of mutually interlaced warp and weft threads. In that case, the pattern of black and white squares in the draft is not the apparent interlacement structure on either side of the weaving. In what follows, we will describe some cases for which a simple criterion does guarantee that the draft directly corresponds to the physical interlacement structure of the fabric.

Recall that a fabric structure is a $k$-harness design if $k$ is the minimum number of harnesses required to weave it; a fundamental block corresponding to a $k$-harness design has exactly $k$ distinct column colorings. All warp threads corresponding to the same column coloring are threaded through the same harness; they rise and fall together as a unit, as the harness rises and falls. The columns of a draft are partitioned into $k$ such units of warp threads, one for each of the $k$ distinct columns. Similarly, weft threads corresponding to identical row colorings in a draft have the same interlacement pattern; we will say they compose a unit of weft threads and note that these units partition the set of all weft strands in the draft. Threads in a single unit, either warp or weft, have identical interlacements and therefore are either in the same set of mutually connected threads or else can be separated from the rest of the weaving.

Lemma 1. If a weaving contains exactly one unit of warp and/or weft, then it separates or "falls apart" into mutually unconnected units of warp and weft.

Proof. Suppose the weaving contains only one unit of warp threads. Because all strands in the unit have the same interlacement structure, any weft thread must either pass over all the warp strands or under all of them. Weft threads that pass over all the warp strands can be lifted off the top, while weft threads that pass under drop off from below. Therefore, the weft threads completely separate from the warp since there are no interlacements at all. Similarly, if a weaving contains only one unit of weft threads, then it separates into individual units of warp and weft.

Lemma 1 leads to the following result:

Theorem 2. If a weaving hangs together, then it is woven with at least two units of warp and at least two units of weft threads. 
Proof. Suppose a weaving hangs together. If it is woven with just one unit of warp or weft, then by Lemma 1 it falls apart, a contradiction.

A corollary of Theorem 2 is intuitively obvious: plain weave is the simplest fabric structure, created with exactly two units of warp and two units of weft. We now prove the following:

Theorem 3. Any weaving can be partitioned into mutually unconnected sets of threads, each set either a fabric that hangs together or a single unit of warp or weft.

Proof. If the entire weaving hangs together, then the theorem is satisfied. Let $W$ denote a set of threads that can be separated from the others. If $W$ hangs together or consists of a single unit of warp or weft, the theorem is satisfied.

Suppose then that $W$ falls apart and consists of two or more units of warp and of weft. We will use proof by induction twice to show that $W$ satisfies the conditions of the theorem.

If $W$ contains exactly two warp units and two weft units, then one warp and/or one weft unit separates from the others. Then by Lemma $1, W$ falls apart into mutually unconnected units of warp and weft, so the theorem is satisfied.

If $W$ contains exactly two warp and three weft units and a warp unit separates from the others, then we can again apply Lemma 1. If a weft unit separates from the others, then two warp and and either one or two weft units remain. If just one weft unit remains, again by Lemma 1 we know the theorem is satisfied. If two warp and two weft units remain, then they either hang together or, as shown in the previous step, fall apart into separate units of warp and weft.

Assume now that the theorem is satisfied by any set of threads with exactly two units of warp and $k$ units of weft, $k \geq 2$.

If $W$ contains exactly two units of warp and $k+1$ units of weft and a single warp separates from the others, then we again apply Lemma 1. If one or more weft units separate from the others, then by the induction assumption, the theorem is satisfied by the thread units that remain. Therefore, the theorem is satisfied for sets $W$ containing two warp units and two or more weft units.

Assume now that the theorem is satisfied by any set of threads having $m$ warp units and two or more weft units, for some $m \geq 2$. If $W$ consists of $m+1$ warp and two or more weft units and falls apart, we remove any weft threads that lift off the top or drop off the bottom. If the remaining set of threads hangs together, we are finished. If this set falls apart, then it must be that at least one warp unit can be separated from the others, with or without weft, leaving subsets with $m$ or fewer warp threads each and, by the induction assumption, the theorem is satisfied.

THE ELECTRONIC JOURNAL OF COMBINATORICS 15 (2008), \#R1 
In later sections, we will consider 3-harness drafts with two or more distinct rows and at least one black and one white square in each row and column. Such a design contains three warp units, corresponding to distinct columns in the draft. The next theorem states that such a draft has from two to six weft units and the resulting fabric hangs together.

Theorem 4. Suppose a drawdown has two or more weft units and each row and column has at least one black and one white square.

If the drawdown has exactly two warp units, then it also has exactly two weft units, and the weaving hangs together.

If the drawdown has exactly three warp units, then it has no more than six weft units, and the weaving hangs together.

Proof. We will prove the theorem for the case that the drawdown has exactly three warp units. The proof for the case of two warp units is similar. Because all the threads in a warp unit rise and fall together, we can without loss of generality assume the draft has exactly three columns, all distinct. Then each row has three squares of black or white. Of the eight ways to color these three positions, six have at least one black and one white square. Therefore, there are six possible weft units.

If a any warp lifts off the top of the weaving, it passes over all weft strands and so its corresponding column in the draft is all black, a contradiction. Suppose a single warp and at least one weft unit can be lifted off the top of the weaving. Since the warp thread cannot pass over all weft strands, at least one weft that is lifted off must pass over this warp, so its corresponding row is all white, a contradiction. A similar argument shows that it is not possible for a single warp, with or without weft units, to drop off the bottom of the weaving.

Suppose a warp thread is in the "middle" of the weaving and not connected with the other two warp strands. Then one of these other two warp strands must lift of the top of the weaving (with or without weft threads) and the other must drop off the bottom. But we just showed this cannot happen. Therefore, the three warp units cannot be separated. Then, if a weft thread lifts off the top of the weaving, it must lift off of all three warp units, so its row is all white, a contradiction. Similarly, a weft thread cannot drop off the bottom of the weaving. Therefore, the weaving hangs together.

Theorem 4 immediately leads to the following corollary:

Corollary 1. If a 2-harness or 3-harness design is generated by a basic block, then the resulting weaving hangs together. 
Proof. By definition, a basic block has at least one black and one white square in each row and column. If the basic block generates a $k$-harness design, then it has exactly $k$ distinct columns or warp units. If $k$ equals 2 or 3 , then by Theorem 4 , the resulting weaving hangs together.

In the sections that follow, we will consider 3-harness designs generated by $m \times 3$ basic blocks having distinct rows and columns. First, we will find out how many of these blocks there are.

\section{Counting $m \times 3$ basic blocks having distinct rows and columns}

Define $B(m, 3)$ as the set of $m \times 3$ basic blocks having $m$ distinct rows and 3 distinct columns, $m>1$. In the following lemma, we show that $m$ must be an integer from 3 to 6 .

Lemma 2. If an $m \times 3$ basic block $b$ has $m$ distinct rows and 3 distinct columns, then $3 \leq m \leq 6$.

Proof. Because $b$ is a basic block, each row and column has at least one black and one white square. If $b$ has two rows, then each column has two squares to be colored in black or white. There are only two ways to color such a column with one black and one white square, so two columns must be identical, a contradiction. Therefore, $m \geq 3$. Since $b$ has three columns, each row has three squares to be colored in black or white. Of the 8 ways to color such a row, 6 use at least one black and one white square. Therefore, $m \leq 6$.

We will need the following result to find the number of elements of $B(m, 3)$.

Lemma 3. Suppose an $m \times 3$ grid of black and white squares has no equal rows and no row is all one color, $3 \leq m \leq 6$. Then no two columns are equal. If $m=3$, no more than one column is all one color. If $m>3$, no column is all one color.

Proof. Suppose two columns are equal, say columns 1 and 2. Since only two colors are used, each column must contain at least two squares the same color. Without loss of generality, suppose the squares in the first two rows of columns 1 and 2 are black. With the first two positions of row 1 both black, the third position must be white since no row is all black. The same is true of row 2, meaning rows 1 and 2 are equal, a contradiction. Therefore, no columns are equal. 
Let $m=3$. Suppose one column is all white and another is all black. Since the remaining column must have at least two squares the same color, the two corresponding rows must be identical, a contradiction. Therefore, if the grid has three rows, no more than one column can be all one color.

Suppose $m>3$ and one column is all one color, say column 1 is all black. There are three ways to color the remaining positions of any row with at least one white square. Since there are more than three rows, at least two rows must be identical, a contradiction. Therefore, if the grid has more than three rows, no column is all one color.

In Theorem 5, we determine the number of elements of $B(m, 3), 3 \leq m \leq 6$.

Theorem 5. There are 84 basic blocks in $B(3,3), 360$ in $B(4,3)$, and 720 in each of $B(5,3)$ and $B(6,3)$.

Proof. Suppose $m=3$. Of the eight ways to color any row in black and/or white, six use at least one black and one white square. Therefore, there are $P(6,3)=120$ blocks with no two rows are alike, where $P(n, k)$ denotes the number of $k$-permutations of $n$ distinct objects. By Lemma 3, we know that no more than one column in any of these 120 blocks is all one color. How many of them have a column that is all one color? Column 1 is all black if one row has black only in the first position, another has black in the first and second positions and the third has black in the first and third positions. These three rows can be arranged in any of 3! ways, so there are six colorings in which column 1 is all black and similarly six in which column 1 is all white. The same applies to columns 2 and 3, so that 36 of the 120 blocks have a column that is either all black or all white. Therefore, there are 84 blocks in $B(3,3)$.

Now suppose $m>3$. There are $P(6, m)$ blocks with no two rows alike and no row all one color. By Lemma 3, all three columns in each of these blocks has at least one white and one black square and therefore is in $B(m, 3)$. Therefore, there the number of basic blocks in $B(m, 3)$ is $P(m, 3)$, which is 360 for $m=4$ and 720 for $m=5$ and 6.

In the next section, we find the number of patterns associated with $B(m, 3)$, $3 \leq m \leq 6$, by counting equivalence classes based on row and column translations of a defining block.

\section{Patterns unique under row/column translations}

How many different patterns or fabric structures are associated with $B(m, 3)$ ? To begin, let $m=3$ and consider the design represented in Figure $5 \mathrm{a}$ and the nine basic

THE ELECTRONic JOURNAL OF COMBINATORICS 15 (2008), \#R1 
blocks outlined there.

If a pattern is generated by a $3 \times 3$ basic block, we can identify such a block by placing a $3 \times 3$ grid on the design. Horizontal and vertical translations of this grid generate the same design [27]. A $3 \times 3$ block $b_{1}$ is outlined in the upper left corner of Figure 5a. Outlined to the immediate right of $b_{1}$ is the block $c\left(b_{1}\right)$ that results from sliding the original grid $1(\bmod 3)$ column to the right. Block $c\left(b_{1}\right)$ also results from a cyclic permutation of columns of block $b_{1}$, with column 1 moving to the column 3 position and the other two columns moving one position to the left. We can think of $c$ as a function from $B(3,3)$ to itself. Since $c$ is one-to-one, it is a permutation [17] of $B(3,3)$.

Block $c c\left(b_{1}\right)=c\left(c\left(b_{1}\right)\right)$ outlined in the upper right of Figure 5 a results from sliding the original grid $2(\bmod 3)$ columns to the right. Sliding the grid $3(\bmod 3)$ positions to the right, we find the original block $b_{1}$, so $\operatorname{ccc}\left(b_{1}\right)=b_{1}=i\left(b_{1}\right)$ where $i$ denotes the identity permutation. Similarly, sliding the original grid in Figure 5a down one or two positions is equivalent to making a cyclic permutation of rows of block $b_{1}: r\left(b_{1}\right)$ and $\operatorname{rr}\left(b_{1}\right)$, respectively. Composition of row and column translations results in the four remaining basic blocks in Figure 5a. Since composition of row and column translations is commutative, the nine row/column translation permutations $i, c, c c, r, r r, c r, c r r, c c r, c c r r$ compose a permutation group $G(3,3)$ of the set $B(3,3)$. A pattern corresponds to an equivalence class of basic blocks under the permutation group of row/column translations. The equivalence class for the pattern in Figure 5a contains nine basic blocks of $B(3,3)$, one for each of the permutations in $G(3,3)$.

Consider now the pattern in Figure 6a with its corresponding basic blocks outlined. This structure is an example of a regular or simple twill: shifting the colorings in any row one position to the right (as in this case for a right twill) or the left (for a left twill) gives the colorings of the row below it [2]. The basic block $b_{2}$ outlined at the top left of Figure 6a defines the 3-harness right twill pattern, as does each of the other $3 \times 3$ blocks outlined in the figure. The equivalence class for the right twill pattern in Figure 6a contains three distinct blocks of $B(3,3)$ and for each of these blocks $b, b=r c(b)=\operatorname{rrcc}(b)$. That is, each right twill block is invariant under the permutations $r c$ and $r r c c$. In general, a block $b$ is invariant under a permutation $g$ and the pair $(g, b)$ is an invariance if $b=g(b)$. The equivalence class for the design in Figure $6 \mathrm{a}$ is associated with the nine invariances $\left(i, b_{2}\right),\left(r c, b_{2}\right),\left(r r c c, b_{2}\right),\left(i, c\left(b_{2}\right)\right)$, $\left(r c, c\left(b_{2}\right)\right),\left(\operatorname{rrcc}, c\left(b_{2}\right)\right),\left(i, c c\left(b_{2}\right)\right),\left(r c, c c\left(b_{2}\right)\right),\left(\operatorname{rrcc}, c c\left(b_{2}\right)\right)$.

The equivalence class of Figure $5 \mathrm{a}$ is associated with the nine invariances $\left(i, b_{1}\right),\left(i, c\left(b_{1}\right)\right),\left(i, c c\left(b_{1}\right)\right),\left(i, r\left(b_{1}\right)\right),\left(i, r c\left(b_{1}\right)\right),\left(i, r c c\left(b_{1}\right)\right),\left(i, r r\left(b_{1}\right)\right),\left(i, \operatorname{rrc}\left(b_{1}\right)\right)$, $\left(i, \operatorname{rrcc}\left(b_{1}\right)\right)$. In general, each equivalence class of $B(3,3)$ is associated with a set of nine invariances and these sets form a partition of the collection of all invariances 


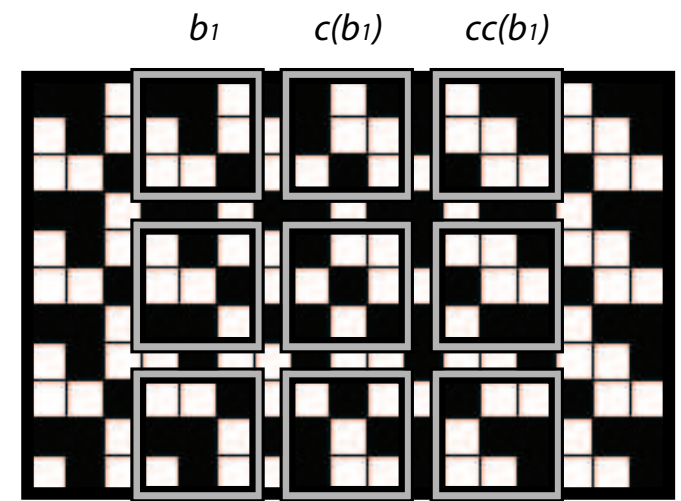

(a) pattern generated by $b_{1}$

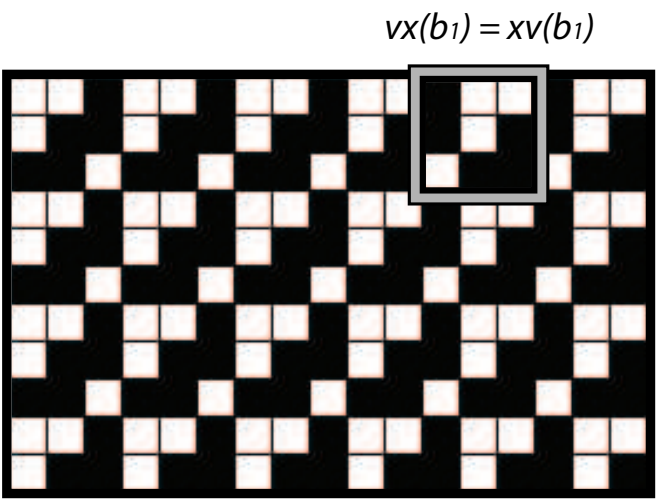

(b) reverse side

Figure 5: (a) Fabric structure defined by the $3 \times 3$ basic block $b_{1}$ outlined in the upper left. The remaining blocks correspond to row/column translations of $b_{1}$. Labeling from left to right, the basic blocks in the first row are $b_{1}, c\left(b_{1}\right)$ and $c c\left(b_{1}\right)$; in the second row, $r\left(b_{1}\right), \operatorname{rc}\left(b_{1}\right)$ and $\operatorname{rcc}\left(b_{1}\right)$; and in the third row, $\operatorname{rr}\left(b_{1}\right), \operatorname{rrc}\left(b_{1}\right)$ and $\operatorname{rrcc}\left(b_{1}\right)$. (b) Reverse of the fabric in (a), defined by the block outlined in the upper right.

under row/column translations. Therefore, the total number of invariances equals nine (the number of permutations) times the number of equivalence classes. If we can find the number of invariances, we will know the number of equivalence classes or designs. This is a special case of a theorem known as Burnside's Lemma ( [17], page 136; [26], page 95), stated below:

Burnside's Lemma Let $G$ be a permutation group of a set $S$. The number of equivalence classes of $S$ induced by $G$ equals the total number of invariances $(g, s)$ divided by the number of permutations in $G$, where $g \in G$ and $s \in S$.

We use Burnside's Lemma now to prove that under row/column permutations, there are twelve equivalence classes of patterns generated by blocks in $B(3,3)$.

Lemma 4. There are 12 patterns associated with $B(3,3)$.

Proof. By Theorem 5, there are 84 invariances of the form $(i, b)$ where $b \in B(3,3)$. Because no block in $B(3,3)$ has equal rows or equal columns, there are no invariances of the form $(r, b),(r r, b),(c, b)$ or $(c c, b)$. Consider the permutation $r c$. Letting $(\mathrm{i}, \mathrm{j})$ denote the color in the (row $\mathrm{i}$, column $\mathrm{j}$ ) position of a basic block $b$, we denote the colorings of $b$ and $r c(b)=c(r(b))$ as follows: 


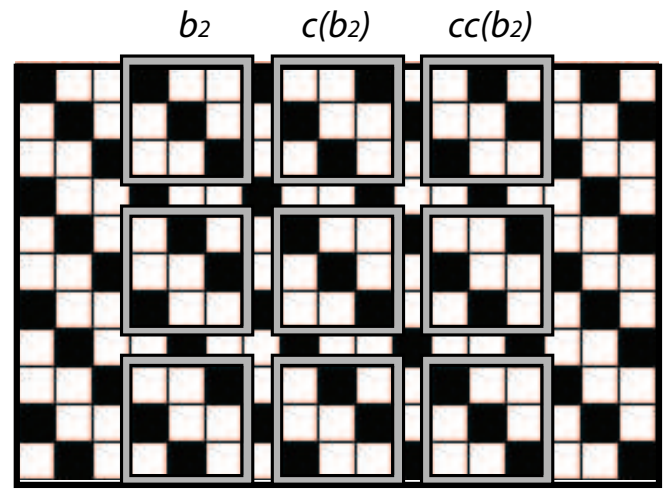

(a) (b)

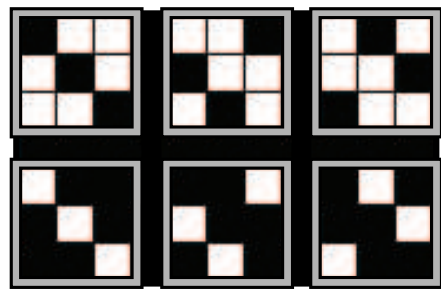

(c)

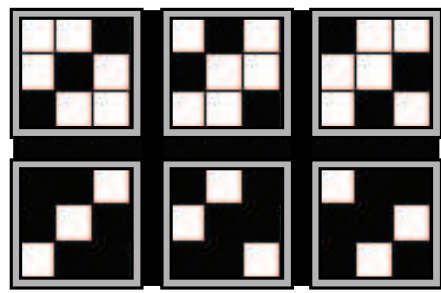

Figure 6: (a) The right twill fabric structure defined by the $3 \times 3$ basic block $b_{2}$ outlined in the upper left of the draft. The remaining blocks correspond to row/column translations of $b_{2}$. Labeling from left to right, the basic blocks in the first row are $b_{2}=r c\left(b_{2}\right)=\operatorname{rrcc}\left(b_{2}\right), c\left(b_{2}\right)=r c c\left(b_{2}\right)=\operatorname{rr}\left(b_{2}\right)$ and $c c\left(b_{2}\right)=r\left(b_{2}\right)=\operatorname{rrc}\left(b_{2}\right)$. (b) The 6 basic blocks invariant under $r c$ and $\operatorname{rrcc}$ are right twills. (c) The 6 basic blocks invariant under $r r c$ and $r c c$ are left twills. 


$$
b=\left(\begin{array}{lll}
(1,1) & (1,2) & (1,3) \\
(2,1) & (2,2) & (2,3) \\
(3,1) & (3,2) & (3,3)
\end{array}\right) \quad r c(b)=\left(\begin{array}{ccc}
(2,2) & (2,3) & (2,1) \\
(3,2) & (3,3) & (3,1) \\
(1,2) & (1,3) & (1,1)
\end{array}\right)
$$

We see that $b$ is invariant under $r c$ if the color in the $(\mathrm{i}, \mathrm{j})$ position of $b$ is in the $(\mathrm{i}-1, \mathrm{j}-1)(\bmod 3)$ position of $r c(b)$, so the color is constant within the three right diagonals (upper left to lower right). Any diagonal can be either black or white, but the three diagonals cannot all be the same color (or the block would be all one color); therefore, there are six blocks that are invariant under $r c$. These six blocks, shown in Figure 6b, are right twill designs. Note that the blocks in the second row are color reversals of the blocks in the first row. These same six blocks are invariant under the permutation $\operatorname{rrcc}$ that moves the color in the $(\mathrm{i}, \mathrm{j})$ position of $b$ to the $(\mathrm{i}-2, \mathrm{j}-2)$ (mod 3) position of $\operatorname{rrcc}(b)$.

The permutation $r c c$ corresponds to sliding a $3 \times 3$ grid on a pattern down one row position and to the right two column positions, equivalent to sliding one row down and one column to the left. The color in the (i, j) position of a block $b$ is in the $(\mathrm{i}-1, \mathrm{j}-2)=(\mathrm{i}-1, \mathrm{j}+1)(\bmod 3)$ position of $\operatorname{rcc}(b)$. Therefore, a block $b$ is invariant under $r c c$ if the color is constant within the three left diagonals (upper right to lower left). The six blocks that are invariant under $r c c$, as well as $r r c$, correspond to left twill designs, shown in Figure 6c.

Each of the four compositions $r c, r c c, r r c$ and $r r c c$ has six invariances. These 24 invariances, combined with the 84 identity invariances, give the 108 invariances of $B(3,3)$ under the 9 row/column translations. By Burnside's Lemma, therefore, there are 12 equivalence classes under these translations.

We now prove that under row/column permutations, there are 30 equivalence classes of patterns generated by blocks in $B(4,3)$ and 48 generated by blocks in $B(5,3)$.

Lemma 5. There are 30 patterns associated with $B(4,3)$ and 48 associated with $B(5,3)$.

Proof. By Theorem 5, there are 360 basic blocks in $B(4,3)$ and 720 in $B(5,3)$.

Consider first patterns associated with $B(4,3)$. There are twelve elements in the permutation group $G(4,3)$ of row/column translations of $B(4,3)$. Is there a basic block $b$ in $B(4,3)$ that is invariant under a row/column translation other than the identity? There are no invariances under simple row translations because no rows of $b$ are equal and similarly no invariances under simple column translations. If $b$ 
were invariant under $r c$, then any three consecutive rows would represent a right twill pattern and this can only happen if all rows are the same color, a contradiction. Similarly, $b$ is not invariant under $r c c$, because then any three consecutive rows would represent a left twill pattern that again can only happen if the entire block is the same color. A similar argument shows that there are no invariances under rrrc and rrrcc. Invariance under $r r c$ or $r r c c$ implies that all the squares in rows 1 and 3 are the same color and likewise for rows 2 and 4 , a contradiction. Therefore, there are 360 invariances under row/column translations, all of the form $(i, b)$ where $b$ is in $B(4,3)$ and $i$ is the identity permutation. Applying Burnside's Lemma, we see that there are 30 equivalence classes (or patterns) of $B(4,3)$ under row/column translations.

The permutation group $G(5,3)$ of row/column translations of $5 \times 3$ blocks has fifteen elements. By an argument similar to the $4 \times 3$ case, we know that the only $5 \times 3$ blocks invariant under a permutation in $G(5,3)$ other than the identity are the two blocks that are all one color and they are not members of $B(5,3)$. Therefore, the only invariances under row/column translations are of the form $(i, b)$ were $b$ is in $B(5,3)$ and $i$ is the identity permutation, and there are 720 of these. Then by Burnside's Lemma, we see that there are 48 designs associated with $B(5,3)$.

More proof is required to show that under row/column permutations there are 44 equivalence classes of patterns associated with $B(6,3)$. The reason is that, as in the $3 \times 3$ case, there are invariances under permutations other than the identity.

Lemma 6. There are 44 patterns associated with $B(6,3)$.

Proof. There are eighteen row/column permutations in $G(6,3)$. Let $b$ be a block in $B(6,3)$. If $b$ is invariant under $r c$, then rows 1 and 4 are equal, as are row 2 and 5 , and rows 3 and 6 , a contradiction since blocks in $B(6,3)$ have distinct rows. Similarly, if $b$ is invariant under any permutation other than $r r c, \operatorname{rrrrc}, \operatorname{rrcc}$ and rrrrcc, then some rows are equal, a contradiction. For $b$ to be invariant under rrc or $\operatorname{rrrcc}$, there must be a right twill in odd numbered rows and another right twill (the color inverse of the first) in even numbered rows, as in $b_{24}$ of Figure 7 . To see how many such blocks there are, we note that the first row can have either one or two black squares. After that selection, there are three ways to start the twill that begins in row 1 and three ways to start its color inverse in row 2 . Therefore, there are 18 blocks in $B(6,3)$ invariant under $r r c$ and rrrrcc. If $b$ is invariant under $r r c c$ or $\operatorname{rrrc}$, then there must be a left twill in odd numbered rows and its color inverse in even numbered rows, and there are 18 such blocks. By Theorem 5, there are 720 $b$ in $B(6,3)$ and therefore 720 invariances of the form $(i, b)$. There are 18 invariances under each of the permutations $r r c, r r r r c c, r r c c$ and $r r r r c$. Therefore, there are 792 

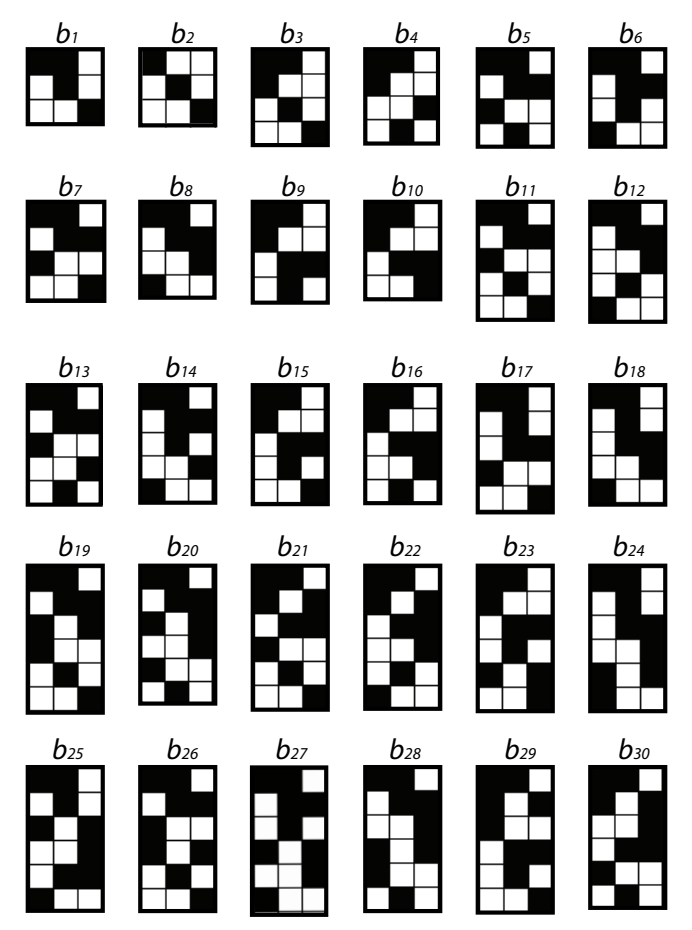

Figure 7: Thirty basic blocks of size $3 \times 3$ through $6 \times 3$.

invariances associated with the 18 permutations of $G(6,3)$. Therefore, by Burnside's Lemma, there are 44 patterns associated with $B(6,3)$.

We summarize the results of this section in the following theorem:

Theorem 6. For $B(3,3), B(4,3), B(5,3)$ and $B(6,3)$, respectively, the number of patterns or equivalence classes under row/column translations is 12, 30, 48 and 44 .

The next step is to discuss how these patterns can be grouped into design families based on weaving symmetries. We will find it helpful to refer to the basic blocks shown in Figure 7. With the exception of $b_{2}$, all of the blocks have at least one row with two black squares; since row and column translations of a block do not change the pattern generated, we are free to consider these blocks as having black squares in the first two positions of the first row, as in Figure 7. This restriction will be useful in counting design families in the following sections.

We begin with weaving symmetries associated with $B(3,3)$. 


\section{$6 \quad$ Weaving symmetries}

Consider the $11 \times 17$ design in Figure $5 \mathrm{a}$ as a piece of woven fabric and imagine turning it over, with top and bottom positions maintained, to see the reverse side represented by the $11 \times 17$ design in Figure $5 \mathrm{~b}$. Turning the fabric over reverses the order of the columns in the original design. Also, because warp threads that show on the face of the fabric are hidden under weft threads on the reverse (and vice versa), the colors must be exchanged. To achieve this "reverse side" directly, the weaver threads warp yarns through the harnesses in reverse order and lifts, for each weft yarn, only the warp threads that were not lifted in the original draft.

The $3 \times 3$ basic block outlined in Figure 5 b defines the "reverse" side of the pattern in Figure $5 \mathrm{a}$, which is generated by the block $b_{1}$. To obtain this new block from $b_{1}$, we reverse the order of the columns and exchange colors. Let $v$ denote the operation that reverses the order of the columns of a block (reflecting across the vertical axis) and $x$ the operation that exchanges black and white. Then the outlined block in Figure $5 \mathrm{~b}$ is $v x\left(b_{1}\right)=x v\left(b_{1}\right)$.

Reversing the order of the lifting sequence corresponds to the operation $h$ that reverses the order of the rows of a block (reflecting over the horizontal axis). The composition $h v$ is equivalent to rotating a block 180 degrees.

Noting that the operations $v, h, v h$ and $x$ are commutative, we define weaving symmetries as the operations in the set $W=\{i, v, h, h v, x, v x, h x, h v x\}$. Let $S(3,3)$ denote the set of patterns generated by blocks in $B(3,3)$. Each weaving symmetry is a one-to-one function from $S(3,3)$ to itself and therefore a permutation, and $W$ is a permutation group of $S(3,3)$. A design family contains all patterns that are equivalent under these weaving symmetries. Using the terminology of Grübaum and Shephard ( [8], page 286), patterns in different design families are "essentially distinct", meaning they are geometrically "of different homeomeric types" with respect to symmetry.

The pattern in Figure 5a and its reverse in Figure 5b are members of the nontwill design family of $B(3,3)$, shown in Figure 8a. There are eight patterns in this design family, generated by $w\left(b_{1}\right)$, where $w \in W$.

Figure $8 \mathrm{~b}$ shows the weaving symmetries for the twill design family of $B(3,3)$. There are four unique patterns in this family and each of these patterns is invariant under $h v$. The members of this family are the right and left twills of Figures $6 \mathrm{~b}$ and $6 \mathrm{c}$, respectively. The left twill structure defined by $x v\left(b_{2}\right)=x h\left(b_{2}\right)$ is called jeans twill or denim when used to weave the fabric for blue jeans [2]. Steggall [22] reported two patterns generated by $3 \times 3$ blocks having exactly one black square in each row and column; these patterns are generated by $b_{2}$ and $v\left(b_{2}\right)$. In their article 


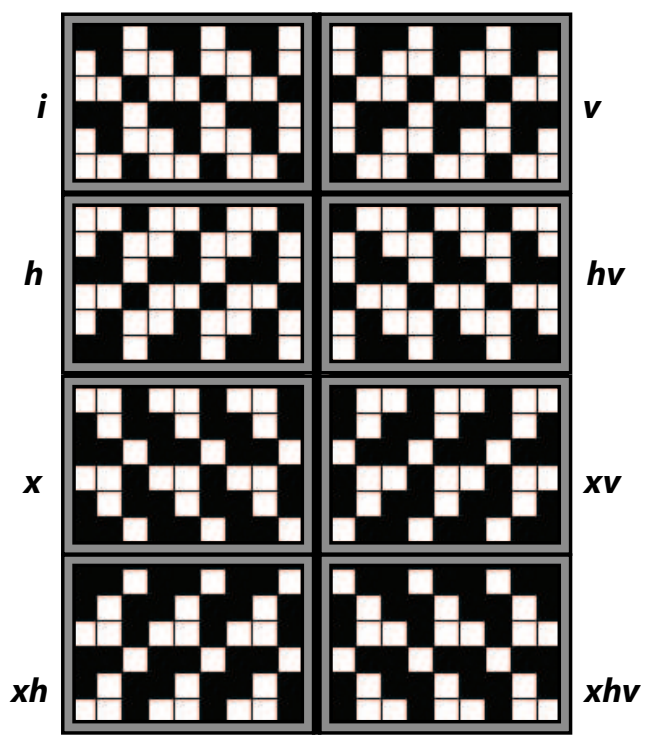

(a) nontwill design family, 8 patterns

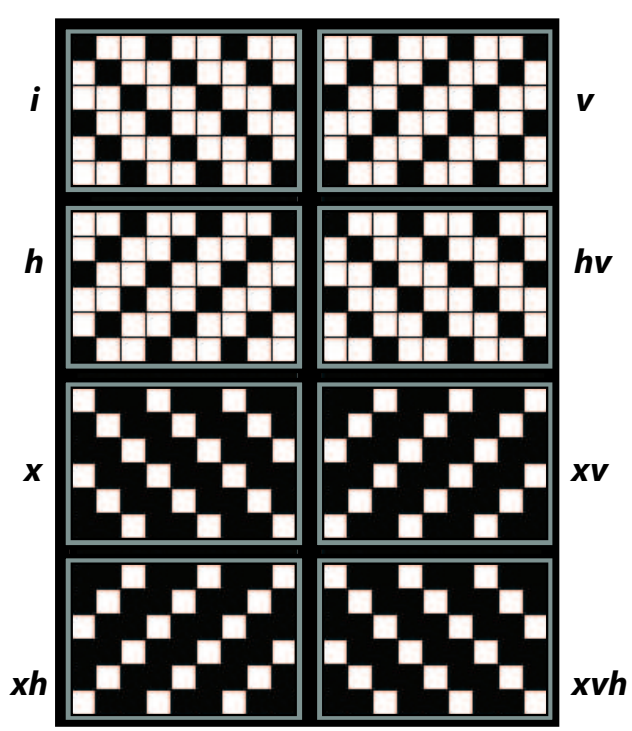

(b) twill design family, 4 patterns

Figure 8: (a) The weaving symmetries of the nontwill design family of $B(3,3)$. The pattern in the upper left is generated by $b_{1}$. The 8 patterns in this family are equivalent to this first pattern under weaving symmetries. (b) The weaving symmetries of the twill design family of $B(3,3)$. The pattern in the upper left generated by $b_{2}=i\left(b_{2}\right)$ is the same as that generated by $h v\left(b_{2}\right)$, so for this family, we say $i=h v$. In the twill design family there are 4 distinct patterns, with $i=h v, v=h, x=x h v$ and $x v=x h$. 
on satins and twills, Grünbaum and Shephard [7] found one "distinct twill" of period 3 , corresponding to the twill design family of $B(3,3)$ represented in Figure $8 \mathrm{~b}$.

Figure 8 shows that the weaving symmetries partition the twelve patterns in $S(3,3)$ into two design families, as stated in Theorem 7 :

Theorem 7. The 12 patterns or fabric structures associated with $B(3,3)$ are partitioned into two design families or equivalence classes under weaving symmetries: the twill family with 4 patterns and the nontwill family with 8 patterns.

For any $b \in B(3,3)$, no row/column translation is equivalent to a weaving symmetry. Therefore, to find the number of design families associated with $B(3,3)$, we can apply Burnside's Lemma to the permutation group $W$ of weaving symmetries of the set $S(3,3)$ of patterns associated with $B(3,3)$. There are 12 invariances of the form $(i, s)$ for $s \in S(3,3)$. There are no invariances under $v$ because this would imply equal columns and similarly, no invariances under $h$. Since there are nine squares in $b$, the number of black squares does not equal the number of white squares $(b$ is not color balanced), so $b$ cannot be invariant under color exchange $x$. Therefore, the only remaining invariances are the four associated with $h v$, as illustrated in Figure 8. Using Burnside's Lemma, we divide the total of 16 invariances by the number 8 of weaving symmetries, to see that there are two equivalence classes of patterns under weaving symmetries.

For $m>3$, there is not always a separation between row/column translations and weaving symmetries. Consider, for example, the $4 \times 3$ blocks $b_{8}$ and $b_{10}$ in Figure 7 . Note that $x\left(b_{8}\right)=\operatorname{rr}\left(b_{8}\right)$ and $v\left(b_{10}\right)=\operatorname{rr}\left(b_{10}\right)$. Rather than considering row/column translations and weaving symmetries together, we will catalog the design families directly, starting with $B(4,3)$.

By Theorem 6, we know that there are 30 patterns associated with $B(4,3)$. We will identify these 30 patterns and classify them into design families based on weaving symmetries.

To find blocks in $B(4,3)$ that correspond to the 30 patterns, we look at possible colorings. Any block in $B(4,3)$ is a $4 \times 3$ grid of black and white squares. Of the twelve squares, the number colored black cannot be less than five or greater than seven because this would result in equal rows or columns. In Lemma 7 , we find the number of patterns associated with blocks having 5, 6 and 7 black squares.

Lemma 7. There are 6 patterns associated with blocks in $B(4,3)$ having 5 black and 7 white squares, 18 patterns with blocks having 6 black and 6 white squares, and 6 patterns with blocks having 7 black and 5 white squares.

Proof. If a block $b$ in $B(4,3)$ has five black squares, then three rows have a single black and one row has two black squares. How many different patterns are associated with 
such a coloring? Since row and column translations of a basic block do not change the pattern, we can without loss of generality suppose columns 1 and 2 of the first row are colored black. There are then six ways to fill each of the remaining three rows with one black square so that no rows or columns are equal. Therefore, there are six patterns associated with blocks having five black and seven white squares. Similarly, there are six patterns that correspond to blocks with seven black and five white squares.

The remaining eighteen patterns must be generated by blocks having six black and six white squares. We can show this directly by noting that any block in $B(4,3)$ having six black and six white squares must have two rows with two black squares and two rows with one black. There are six ways to choose two rows to have two blacks, six ways to fill in those two rows with two blacks and one white, and six ways to fill in the remaining two rows with two whites and one black, for a total of 216 basic blocks. Recall that the only invariances under row/column translations in $B(4,3)$ are with the identity permutation $i$. Applying Burnside's Lemma, we divide the number of invariances (216) by the number of row/column permutations (12), to see that there are 18 patterns generated by blocks having the same number of black and white squares.

We will say that a pattern associated with a basic block having an equal number of black and white squares is color balanced; otherwise, it is color unbalanced. There are no color balanced patterns associated with $B(3,3)$. By Lemma 7 , we know that there are eighteen patterns associated with $B(4,3)$ that are color balanced and twelve that are not.

An unbalanced pattern associated with $B(4,3)$ is generated by a block having either five or seven black squares. A block with seven black squares is the color reversal of a block with five, so for identifying design families, we can consider only blocks with five black squares. Two such blocks are $b_{3}$ and $b_{4}$ of Figure 7 . Accounting for row/column translations and weaving symmetries, these are the only color unbalanced blocks in $B(4,3)$ we need consider.

The twelve unbalanced patterns of $B(4,3)$ are illustrated in Figure 9, separated into two design families based on weaving symmetries. Figure 9a shows the design family associated with the block $b_{3}$ that has black squares in the first two columns of the first row and in columns 1, 2 and 3, respectively, of the remaining rows. There are no invariances under weaving symmetries in this design family, so the family contains eight patterns.

As Figure 9b illustrates, there are four patterns in the design family generated by the block $b_{4}$ that has black squares in the first two columns of the first row and in columns 1, 3 and 2, respectively, of rows 2, 3 and 4 . To denote the pattern invariances 

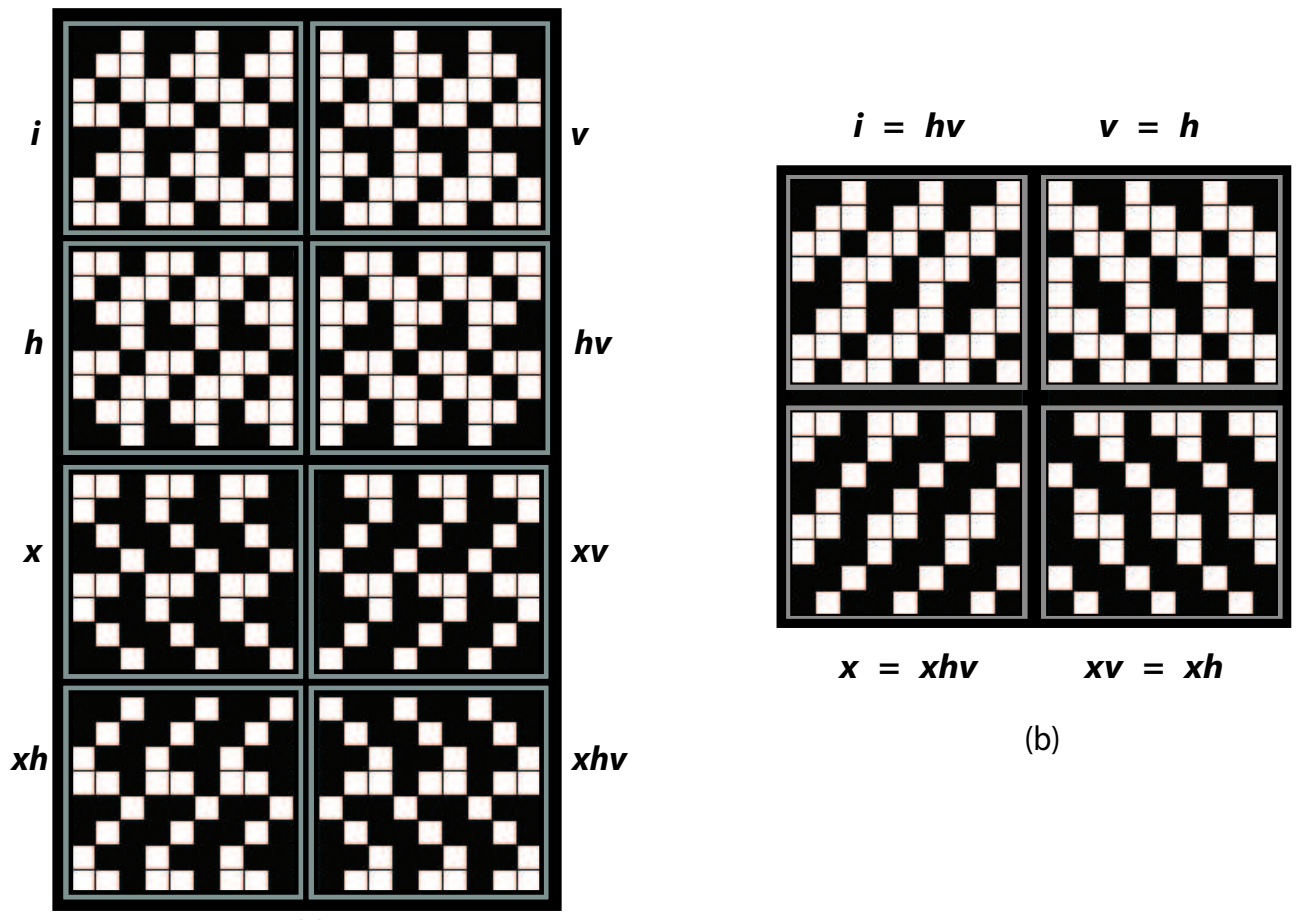

(b)

Figure 9: There are 12 color unbalanced patterns associated with $B(4,3)$ : (a) 8 patterns in the design family generated by $b_{3}$ and (b) 4 patterns in the design family generated by the basic block $b_{4}$. 
in this family, we say $i=h v, v=h, x=x h v$ and $x v=x h$. The patterns in this family have a more twill-like appearance than those of Figure 9a.

The eighteen balanced patterns of $B(4,3)$, illustrated in Figure 10, are partitioned into six design families based on weaving symmetries. Three of these families contain four patterns each and three contain two patterns each.

The blocks $b_{5}-b_{8}$ representing the design families in Figures 10a-d have adjacent rows of double blacks alternating with adjacent rows of single blacks. The blocks $b_{9}$ and $b_{10}$ have alternating rows of double and single blacks and the families associated with them are illustrated in Figures $10 \mathrm{e}$ and $10 \mathrm{f}$.

As Figure 10d shows, a balanced pattern can equal its own color inverse, even though that cannot be true of a basic block. In this case, $x\left(b_{8}\right)=\operatorname{rr}\left(b_{8}\right)$. Also, if a block has no equal columns, then it cannot equal its own vertical reflection. However, as Figure 10f shows, it is possible that such a block generates a pattern that equals its own reflection. In this case, the pattern is generated by $b_{10}$, and $v\left(b_{10}\right)=\operatorname{rr}\left(b_{10}\right)$.

Theorem 8 summarizes these results for $B(4,3)$ :

Theorem 8. There are 30 patterns associated with B(4,3), 18 color balanced and 12 not. These 30 patterns are partitioned into eight design families based on weaving symmetries. Two of these design families contain color unbalanced patterns, one family with eight patterns and the other with four patterns. Of the six design families containing balanced patterns, three contain four patterns each and three contain two patterns each.

We now find the design families associated with $B(5,3)$. Any block in $B(5,3)$ contains five of the six possible colorings of rows with at least one black and one white square, and therefore has either seven or eight black squares. A block with eight black squares is the color reversal of a block with seven, so for identifying design families, we can consider only blocks with seven black squares. A $5 \times 3$ block with seven black squares has two rows of double blacks and three rows of single blacks. The two double rows can be adjacent or separated by rows with single blacks. Taking row/column translations and weaving symmetries into account, we need only consider the $5 \times 3$ blocks $b_{11}-b_{18}$ in Figure 7 .

Figure 11 shows the four design families associated with the blocks $b_{11}-b_{14}$ that have adjacent rows of double blacks. The design families in Figures 11c and 11d have no invariances under weaving symmetries other than $i$ and so contain eight patterns each. There are four patterns in the design families illustrated in Figures 11a and 11b; in each of these families, $i=h v, v=h, x=x h v$ and $x v=x h$.

The four remaining design families associated with $B(5,3)$, shown in Figure 12 , are generated by basic blocks in which the two rows of double blacks are not adjacent. 
(a)

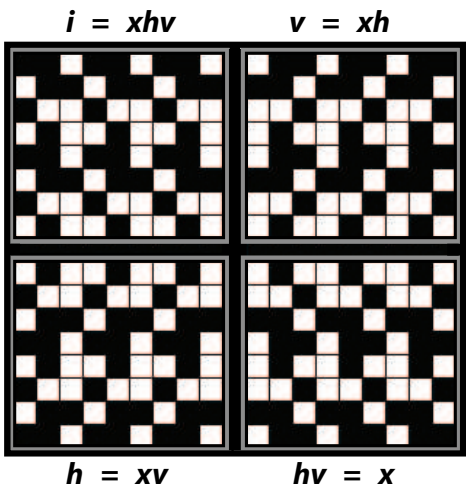

(c)

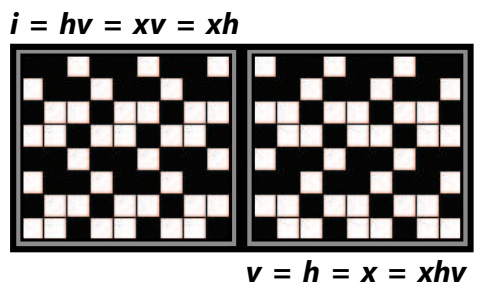

(e)

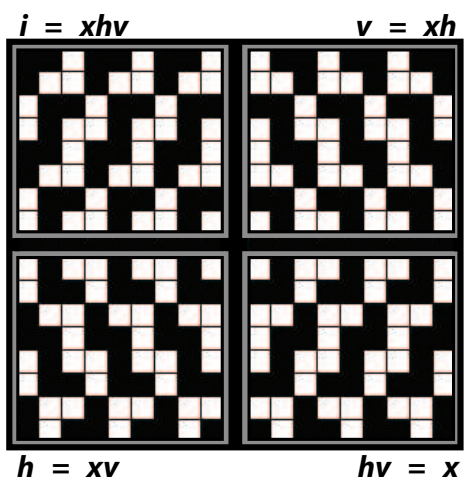

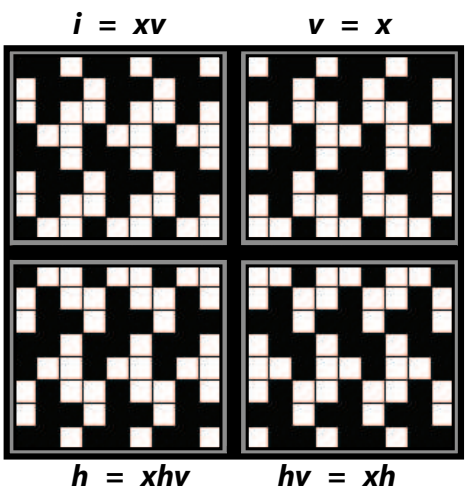

(b)

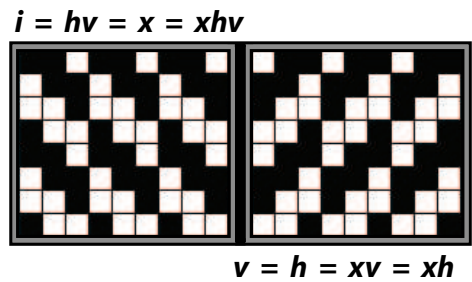

(d)

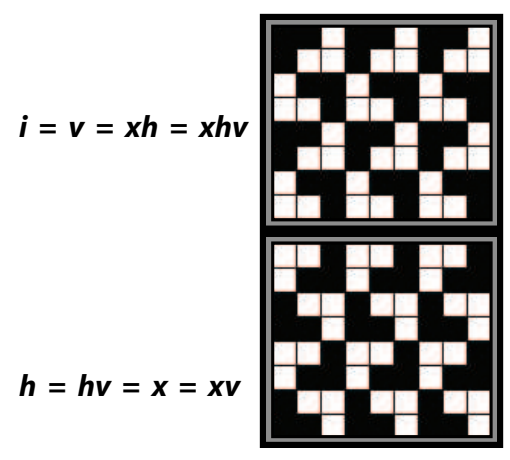

(f)

Figure 10: The design families in (a)-(f) are generated by $4 \times 3$ basic blocks $b_{5}-b_{10}$, respectively. These patterns are all balanced in the sense that the generating blocks have an equal number of black and white squares. 


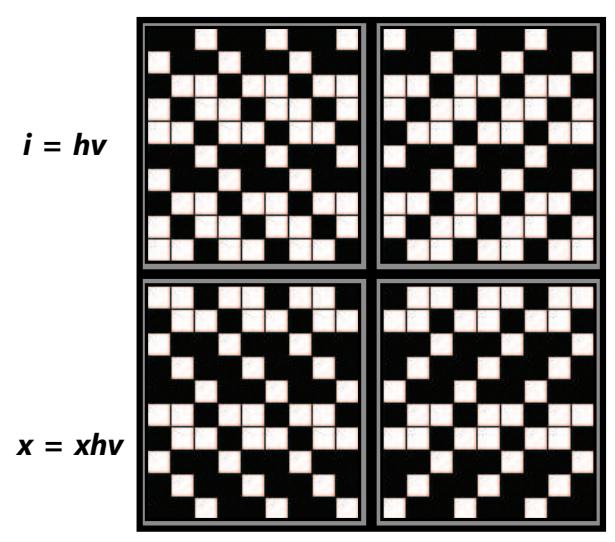

(a)

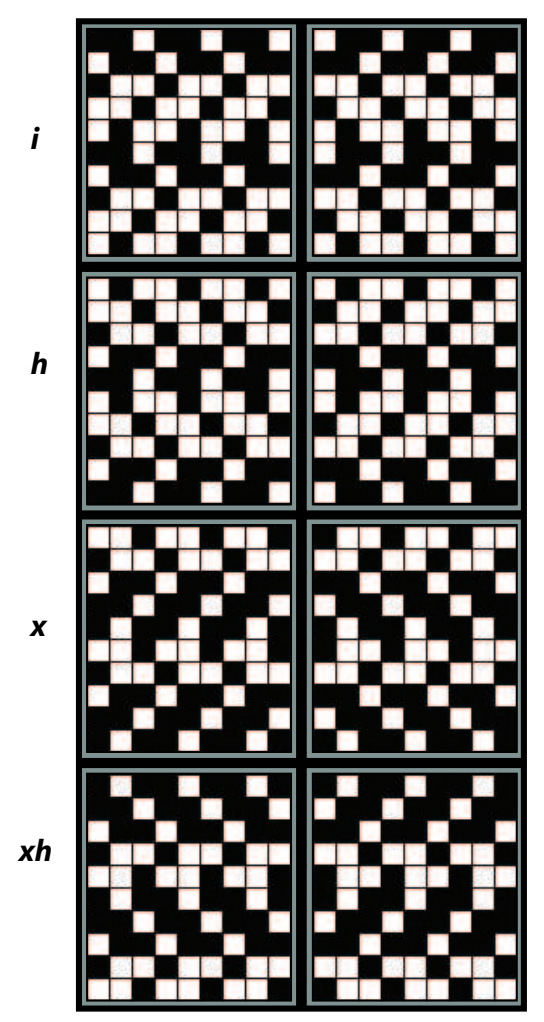

(c)

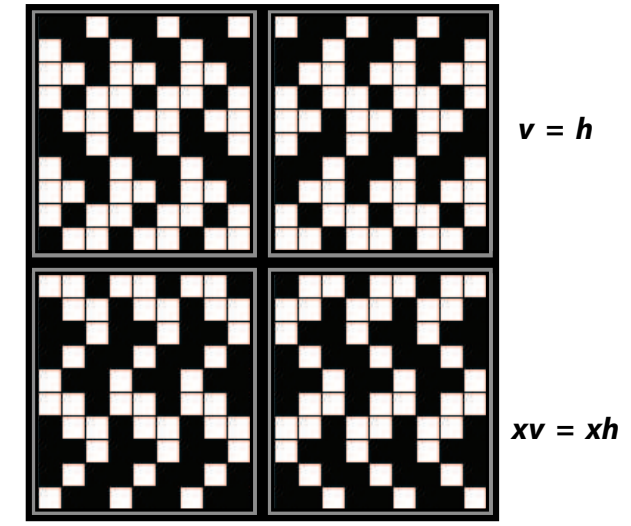

(b)

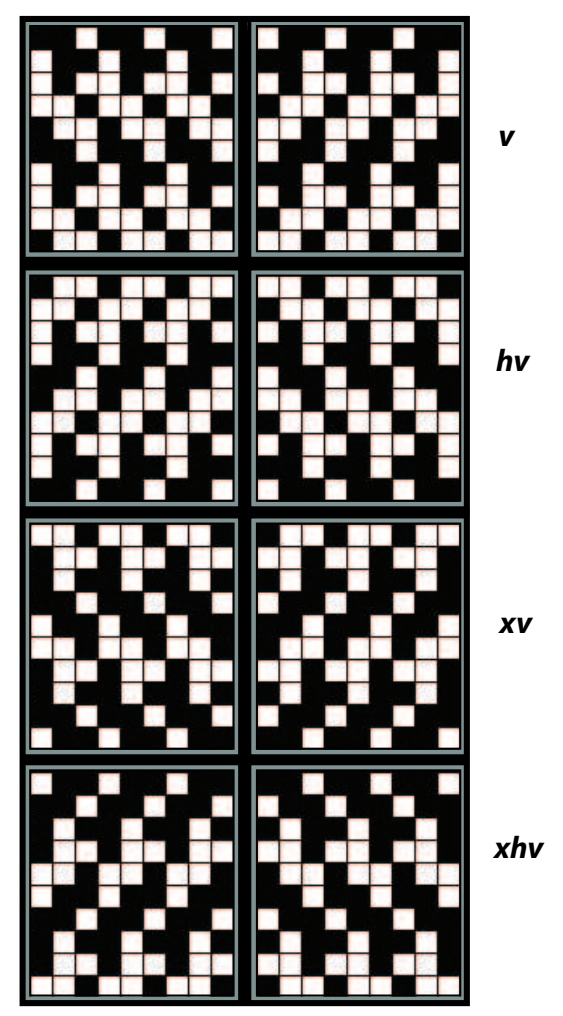

(d)

Figure 11: The design families in (a)-(d) are generated by $5 \times 3$ basic blocks $b_{11}-b_{14}$, respectively. These four basic blocks all have adjacent rows of double blacks. 


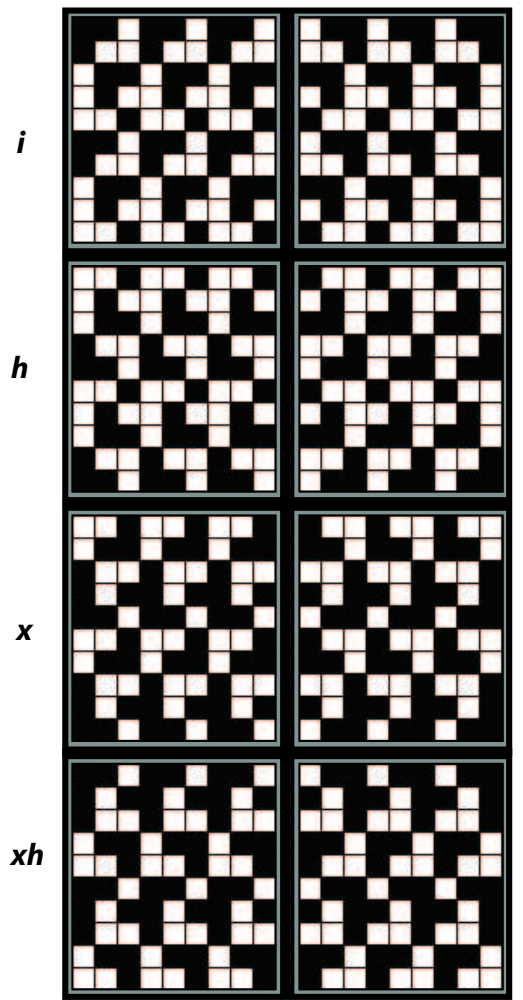

(a)

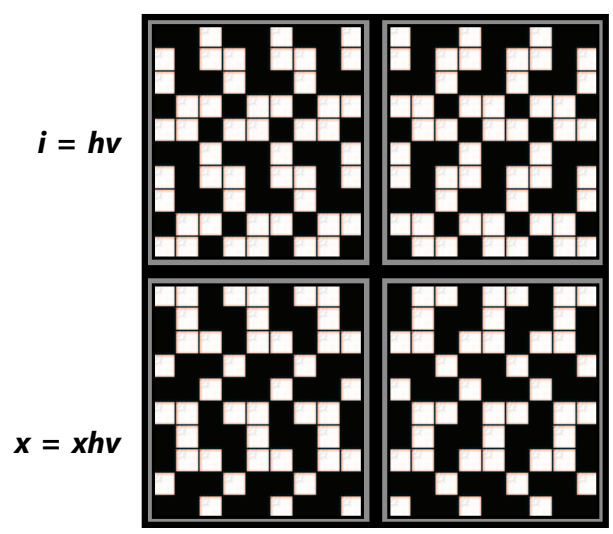

(c)

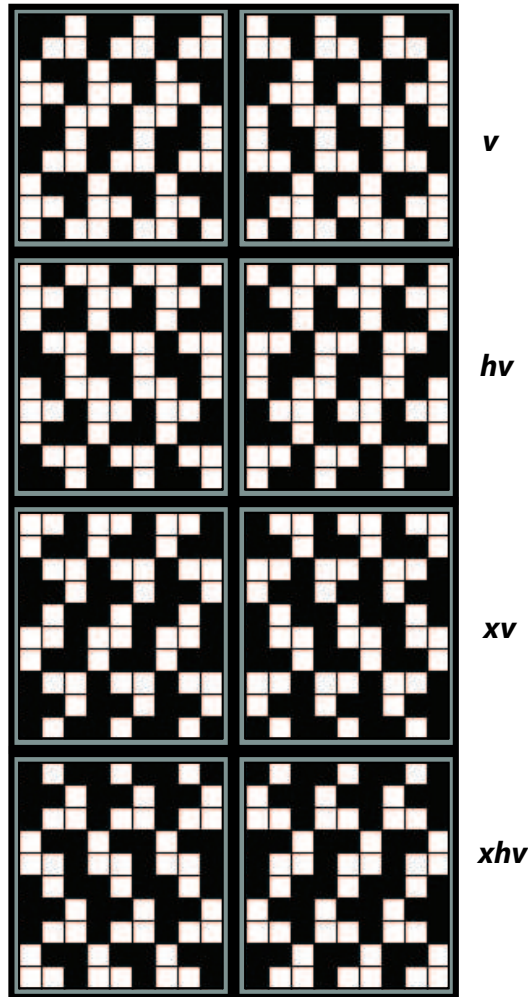

(b)

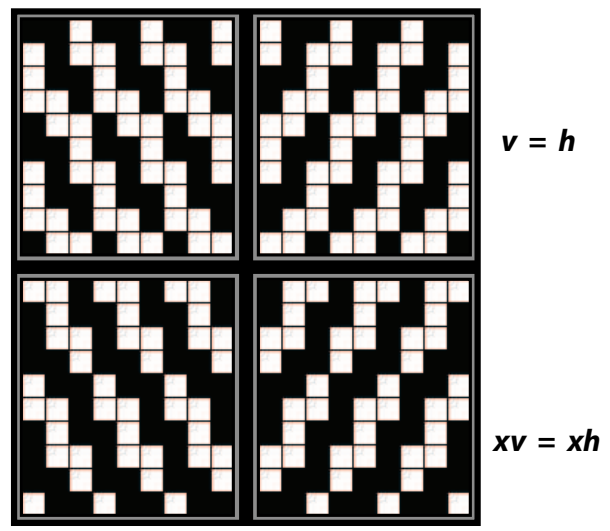

(d)

Figure 12: The design familes in (a)-(d) are generated by the $5 \times 3$ basic blocks $b_{15}-b_{18}$, respectively, that do not have adjacent rows of double blacks. 
The design families in Figures $12 \mathrm{a}$ and $12 \mathrm{~b}$, generated by $b_{15}$ and $b_{16}$, respectively, contain eight patterns each, having no invariances under weaving symmetries other than $i$. The patterns in the design families of Figures $9 \mathrm{c}$ (generated by $b_{17}$ ) and $9 \mathrm{~d}$ (generated by $b_{18}$ ) have the same invariances under weaving symmetries, $i=h v, v=$ $h, x=x h v$ and $x v=x h$, and so these design families have four patterns each. Theorem 9 summarizes these results for $B(5,3)$.

Theorem 9. There are 48 patterns associated with $B(5,3)$, all color unbalanced. These 48 patterns are partitioned into eight design families based on weaving symmetries, four families with eight patterns each and four with four patterns each.

Note that no block with an odd number of squares can be associated with a design family of size 2 since invariance under color reversal requires an equal number of black and white squares. Design families associated with $B(3,3)$ and $B(5,3)$ contain either eight or four patterns. $B(4,3)$, on the other hand, has three design families of four patterns each and three families of two patterns each. We might expect $B(6,3)$ to have a design family with exactly two patterns, and as we will see, there are four such families.

The rows of any block in $B(6,3)$ include all six possible colorings with at least one black and one white. Taking row/column translations and weaving symmetries into account, the only basic blocks we need consider are $b_{19}-b_{30}$ of Figure 7 . These basic blocks have one of these three forms: three adjacent rows of double blacks alternating with three adjacent rows of single blacks as in $b_{19}-b_{22}$ and Figures 13ad; rows of double blacks alternating with rows of single blacks as in $b_{23}-b_{25}$ and Figures 13e-g; and two adjacent rows of double blacks and two adjacent rows of single blacks alternating with one row of each as in $b_{26}-b_{30}$ and Figure 14.

Figure 13 shows twenty patterns organized into seven families. The patterns in the design families in Figure 13a (four patterns), 13b (two patterns) and 13d (four patterns) have three rows of right twill alternating with three rows of right twill or three rows of left twill alternating with three rows of left. The two patterns in the design family of Figure 13c alternate three rows of right twill with three rows of left twill. The two patterns in the design family shown in Figure 13g have left twill in one set of alternate rows and right twill in the other.

The patterns illustrated in Figures 13e and 13f are alternating twills in the sense that alternate rows make up a simple twill pattern, with the two sets of twills going in the same direction. These two design families have very different looks. The patterns in Figure 13e are not at all twill-like, while those in Figure 13f are. Alderman [2] called the patterns in Figure 13f double-faced twills, while Grünbaum and Shephard [7] called them color-alternate twills. In a color-alternate twill, exchanging 
(a)

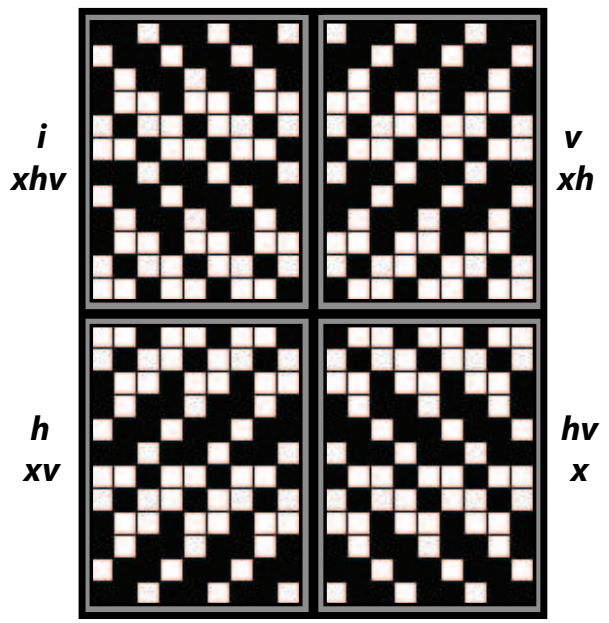

(b)

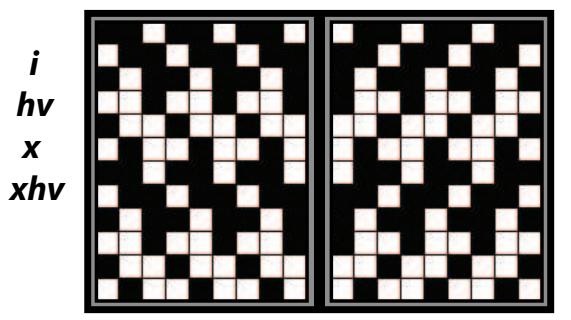

(e)

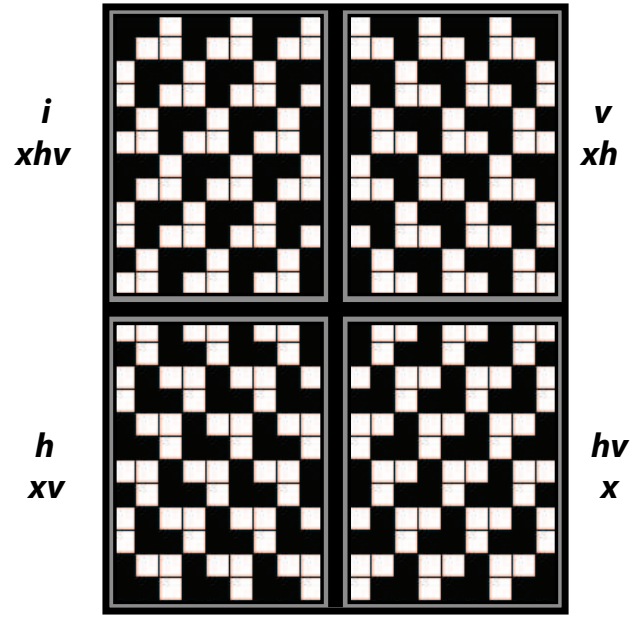

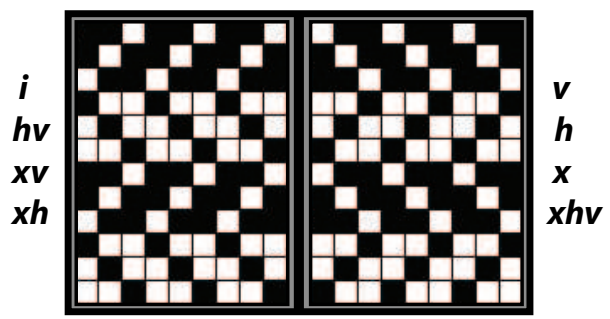

(c)

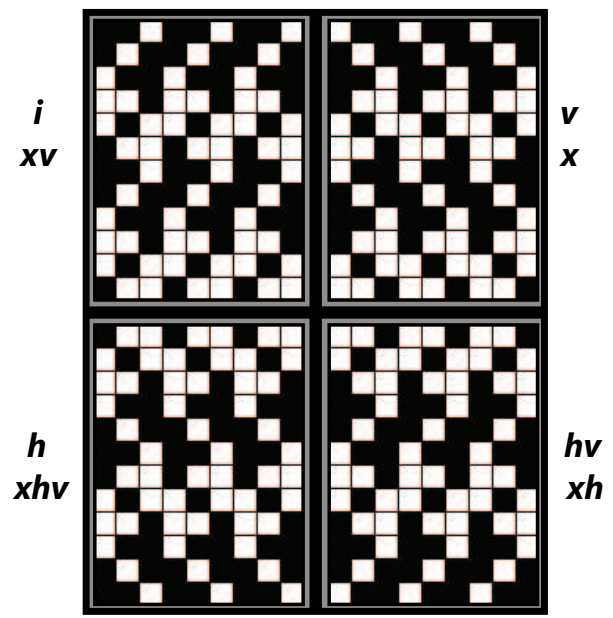

(d)

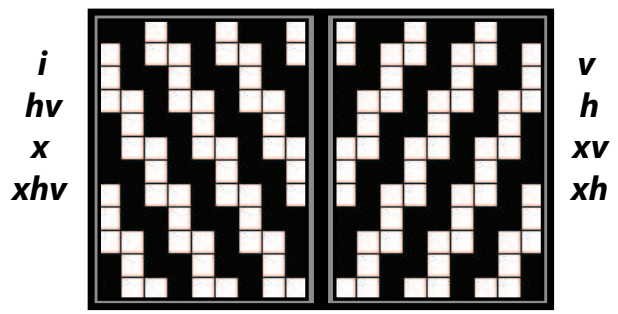

(f)

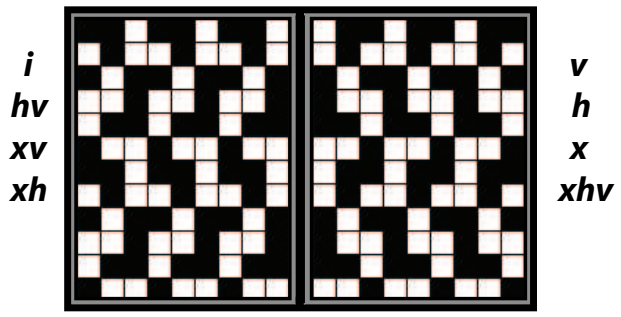

Figure 13: The $6 \times 3$ basic blocks $b_{19}-b_{25}$ generate the design families in (a)-(g), respectively. 
the colorings of any row and shifting one position to the right (the pattern on the right of Figure 13f) or left (the pattern on the left of 13f) gives the row below. The patterns in Figure $13 \mathrm{f}$ are the only color-alternate twills possible on three harnesses.

The remaining five design families of $B(6,3)$, illustrated in Figure 14, include 24 patterns: eight in the design family of Figure 14e and four in each of the design families represented in Figures 14a-d. Theorem 10 summarizes these results for $B(6,3)$.

Theorem 10. There are 44 patterns associated with B6,3), partitioned into 12 design families based on weaving symmetries. One of these families includes eight patterns, seven families have four patterns each and four families have two patterns each.

The results of this and the previous section are presented in the table below. For each basic block size $m \times 3$, the representative blocks of $B(m, 3)$ are listed by color sequence (number of black squares per row). Among the ten color sequences, there are 134 patterns organized into 30 design families based on weaving symmetries.

Some patterns have a twill-like look, with infinite diagonals of black (or white) squares. Others tend to have connected sets of black squares separated from connected sets of white squares. In the next section, we consider the appearance of these tilings of the plane and relate some to polyominoes.

\section{Tiling the plane}

Each of the 30 design families associated with $B(m, 3), 3 \leq m \leq 6$, is represented in Figure 15 by a $4 \times 4$ repetition of one of the basic blocks $b_{1}-b_{30}$ of Figure 7 . The appearance of some of these patterns can be described by geometrical forms created by the black and white squares.

Tilings 2, 4, 18 and 24 have infinite diagonals of edge-wise connected black squares and/or edge-wise connected white squares. The other tilings show distinct shapes created by a finite number of edge-wise connected squares of the same color. A shape made by connecting a collection of unit squares, each square joined to another along an edge, is a polyomino [15]. We can think of the patterns represented in Figure 15 as tilings of the plane in polyominoes.

Pattern 24 is a color-alternate twill with infinite diagonals in each color, alternate diagonals of one color completely separated by diagonals of the other color. 
(a)

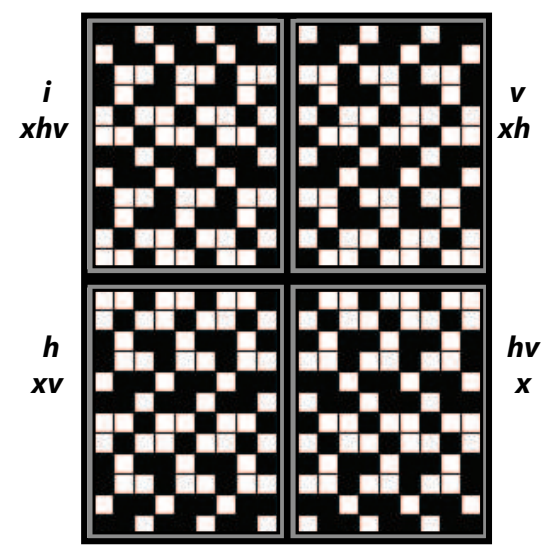

(b)

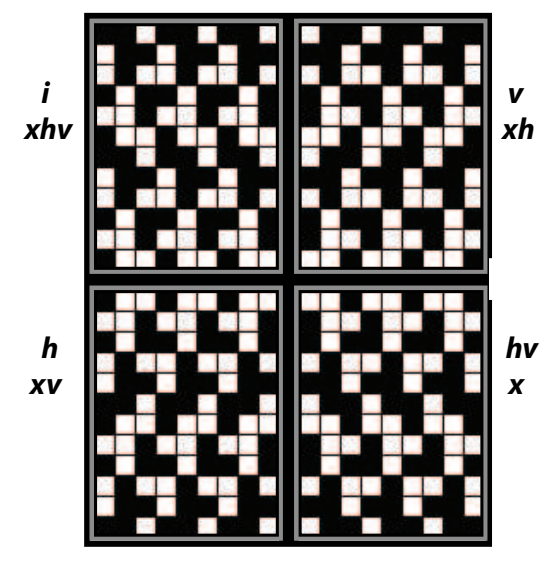

(c)

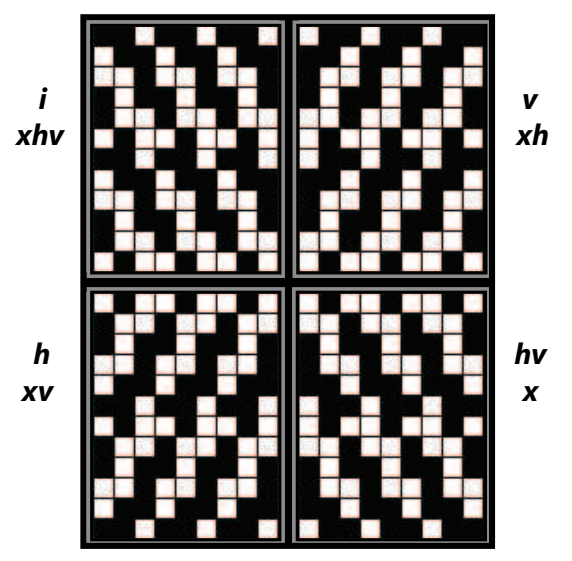

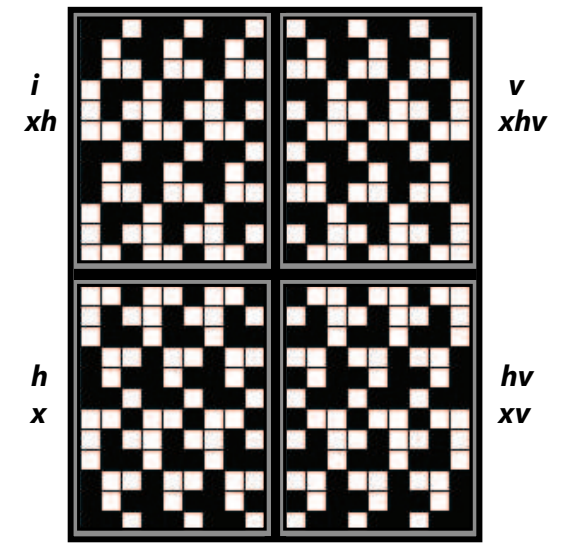

(d)
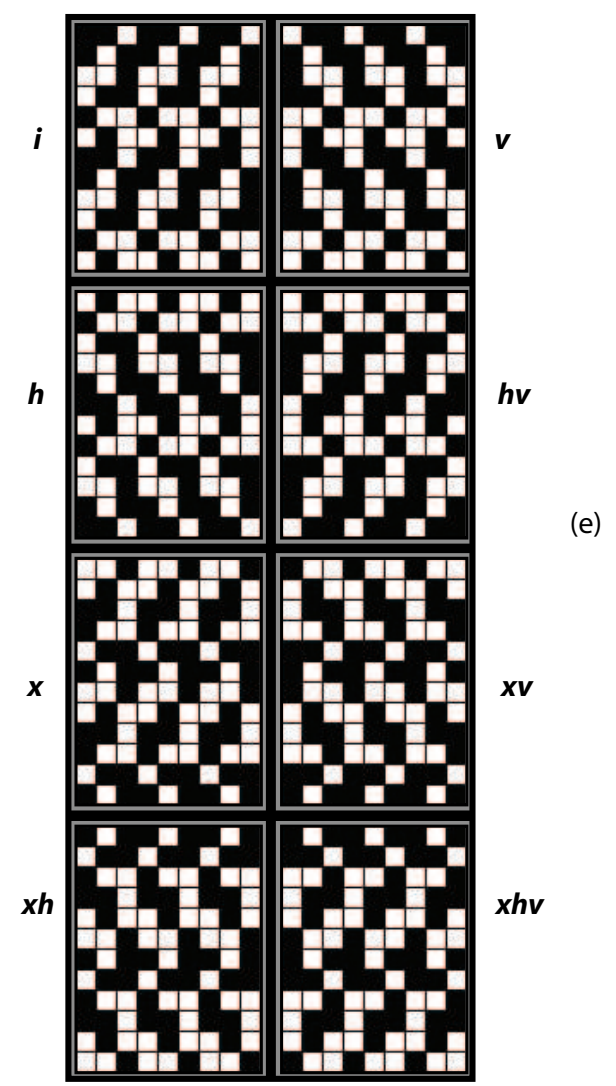

Figure 14: The $6 \times 3$ basic blocks $b_{26}-b_{30}$ generate the design families in Figures 11a-11e, respectively. 
Patterns and designs associated with $B(m, 3), 3 \leq m \leq 6$

\begin{tabular}{|c|c|c|c|c|c|c|}
\hline $\begin{array}{l}\text { Block } \\
\text { size }\end{array}$ & $\begin{array}{l}\text { Black squares } \\
\text { per row of } b_{i}\end{array}$ & $\begin{array}{l}\text { Pattern } \\
i\end{array}$ & $\begin{array}{l}\text { Patterns } \\
\text { in family }\end{array}$ & $\begin{array}{l}\text { Design } \\
\text { families }\end{array}$ & $\begin{array}{r}\text { Total } \\
\text { patterns }\end{array}$ & Figure \\
\hline \multirow[t]{3}{*}{$3 \times 3$} & $2,1,1$ & 1 & 8 & 1 & 8 & $8 \mathrm{a}$ \\
\hline & $1,1,1$ & 2 & 4 & 1 & 4 & $8 \mathrm{~b}$ \\
\hline & & & Total: & 2 & 12 & \\
\hline \multirow[t]{7}{*}{$4 \times 3$} & $2,1,1,1$ & 3 & 8 & 1 & 8 & $9 \mathrm{a}$ \\
\hline & & 4 & 4 & 1 & 4 & $9 b$ \\
\hline & $2,2,1,1$ & 5,6 & 4 & 2 & 8 & $10 a, b$ \\
\hline & & 7,8 & 2 & 2 & 4 & $10 \mathrm{c}, \mathrm{d}$ \\
\hline & $2,1,2,1$ & 9 & 4 & 1 & 4 & $10 \mathrm{e}$ \\
\hline & & 10 & 2 & 1 & 2 & $10 \mathrm{f}$ \\
\hline & & & Total: & 8 & 30 & \\
\hline \multirow[t]{5}{*}{$5 \times 3$} & $2,2,1,1,1$ & 11,12 & 4 & 2 & 8 & $11 a, b$ \\
\hline & & 13,14 & 8 & 2 & 16 & $11 \mathrm{c}, \mathrm{d}$ \\
\hline & $2,1,2,1,1$ & 15,16 & 8 & 2 & 16 & $12 a, b$ \\
\hline & & 17,18 & 4 & 2 & 8 & $12 \mathrm{c}, \mathrm{d}$ \\
\hline & & & Total: & 8 & 48 & \\
\hline \multirow[t]{7}{*}{$6 \times 3$} & $2,2,2,1,1,1$ & 19,22 & 4 & 2 & 8 & $13 \mathrm{a}, \mathrm{d}$ \\
\hline & & 20,21 & 2 & 2 & 4 & $13 \mathrm{~b}, \mathrm{c}$ \\
\hline & $2,1,2,1,2,1$ & 23 & 4 & 1 & 4 & $13 \mathrm{e}$ \\
\hline & & 24,25 & 2 & 2 & 4 & $13 \mathrm{f}, \mathrm{g}$ \\
\hline & $2,2,1,2,1,1$ & 26-29 & 4 & 4 & 16 & $14 a-d$ \\
\hline & & 30 & 8 & 1 & 8 & $14 \mathrm{e}$ \\
\hline & & & Total: & 12 & 44 & \\
\hline
\end{tabular}

Grand total: $\quad 30 \quad 134$

the Electronic Journal of Combinatorics 15 (2008), \#R1 

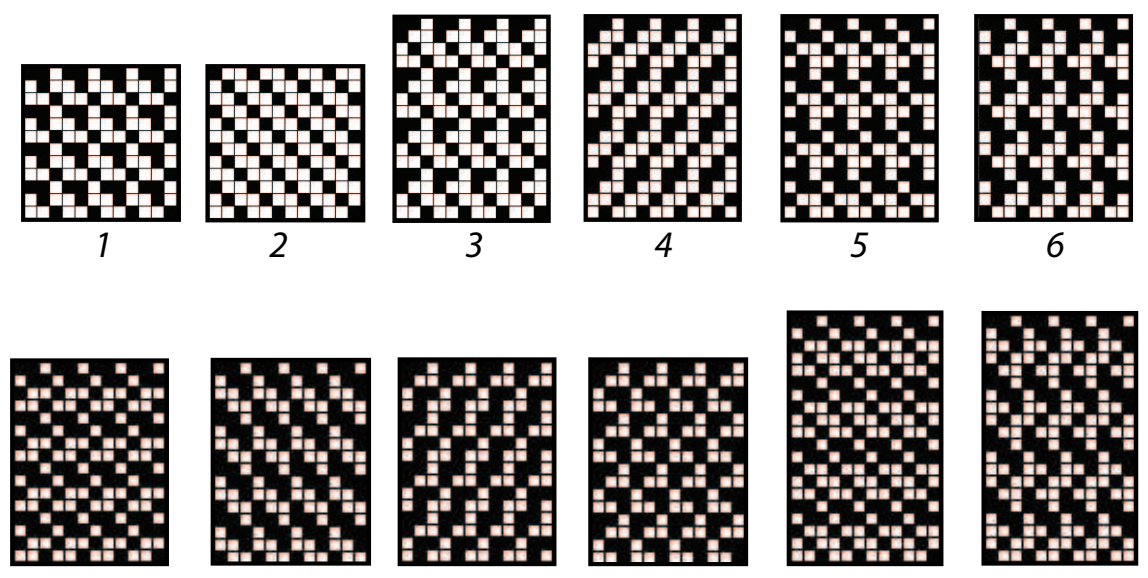

7

$$
8
$$

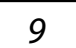

10

11
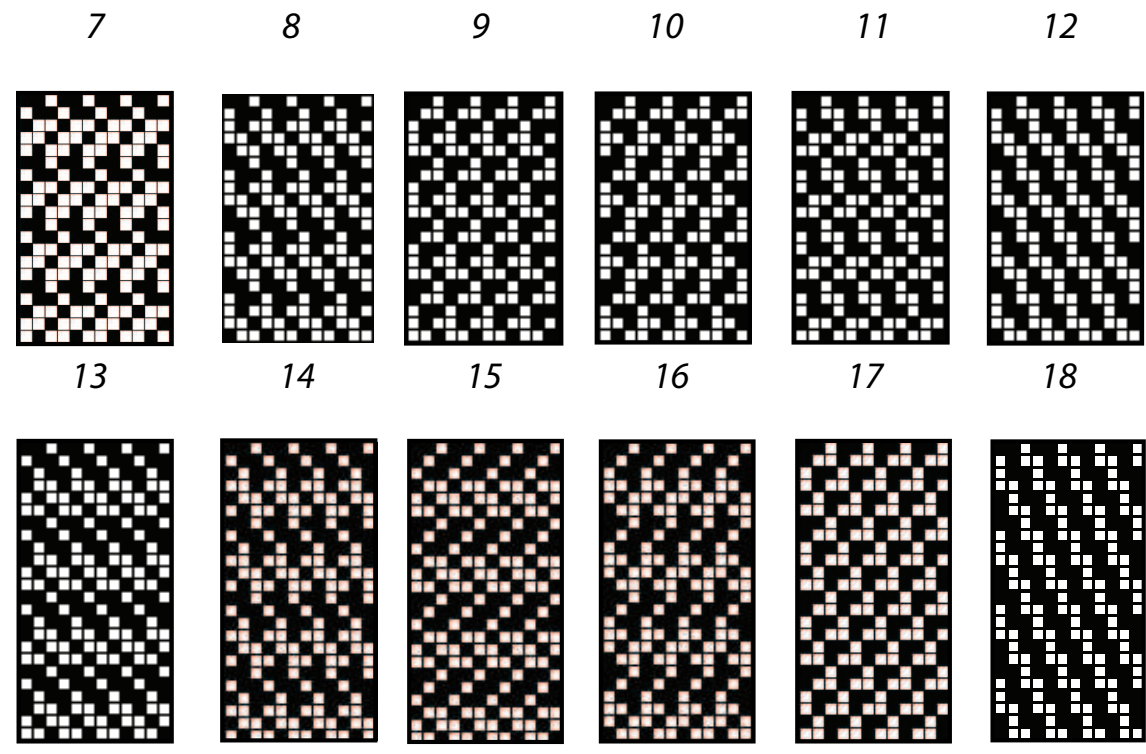

15
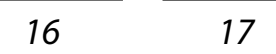

18
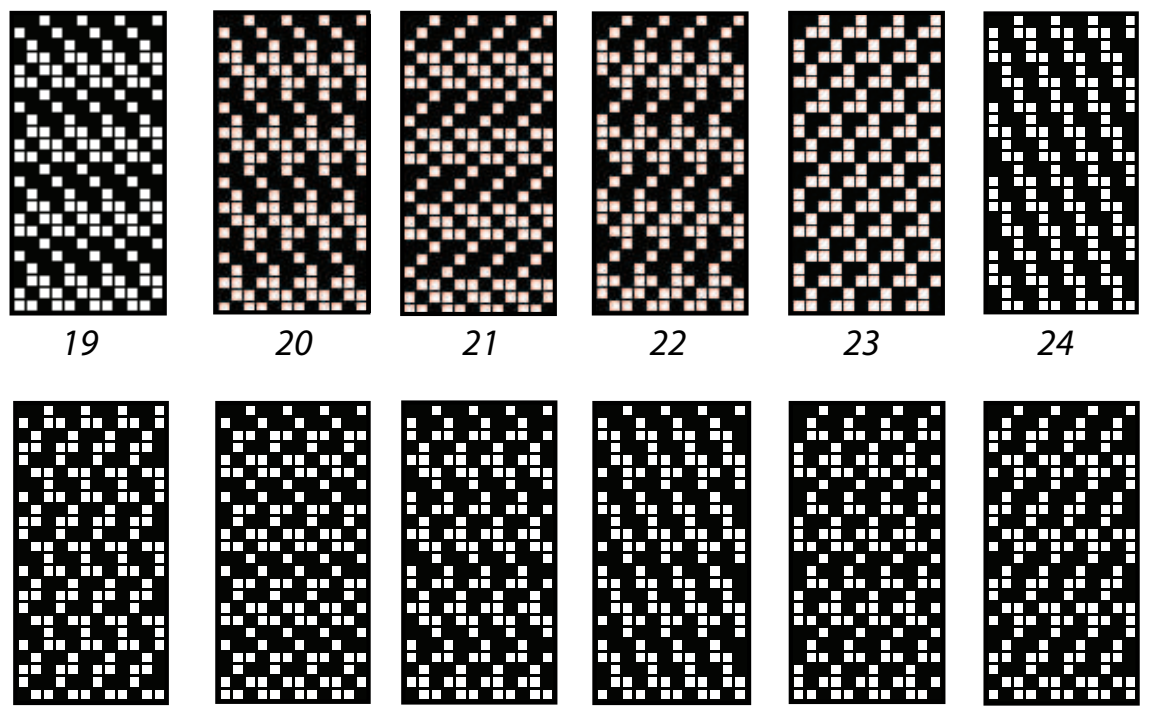

25
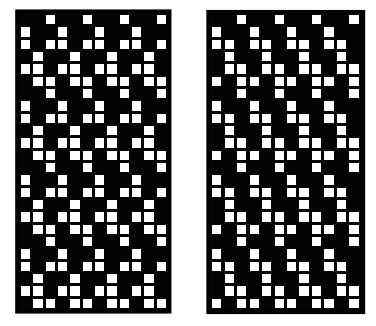

28

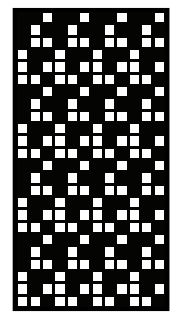

29

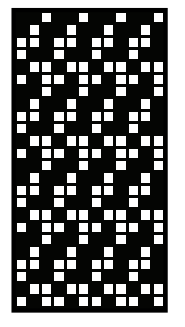

30

Figure 15: Patterns 1-30 are generated by basic blocks $b_{1}-b_{30}$, respectively. 
Each diagonal consists of connected straight trominos, a straight tromino created by connecting three squares in a line. We might call tiling 24 a pure diagonal because squares of each color are connected in infinite diagonals.

In tiling 2, a right twill, we see infinite white diagonals of edge-wise connected dominoes. The black diagonals consist of separate monominoes connected only at corners. This is a mixed pattern because it has infinite diagonals in one color and diagonals of separate polyominoes in the other color. Another mixed pattern is tiling 4, which has infinite white diagonals resulting from repeated edge-wise connections of two dominoes and one straight tromino, and black diagonals of alternating skew tetrominoes and monominoes. The remaining mixed pattern is tiling 18; its infinite white diagonals consist of repeated edge-wise connections of a domino and two straight trominoes and its black diagonals consist of translations of the a single heptomino.

Six patterns in Figure 15 are pure polyomino tilings in the sense that each is created from a single polyomino, translated and possibly rotated or reflected. Tiling 6 is created from a single hexomino in black and vertical reflections of that hexomino in white. Pattern 9 has a hexomino in black and a 180 rotation of that hexomino in white. In pattern 10, vertical reflections of a right tromino appear in both black and white, while tiling 23 contains a right tromino in black and its 180 degree rotation in white. Tiling 28 has a nonomino (nine squares) in black and its 180 degree rotation in white. Tiling 8 is the "purest" pure polyomino in that it consists of simple tranlations of a single hexomino in black and white. Each of the other tilings in Figure 15 is a multiple polyomino tiling since at least two different polyominoes make up the pattern.

Many of the polyomino problems discussed in Golomb [15] and Martin [19] involve tiling a region such as a rectangle with polyminoes. In this work, we proceeded in the other direction by creating tilings of the plan from rectangles of black and white squares and then noting how the resulting patterns could be related to polyominoes.

\section{Discussion}

Each of the patterns discussed in this paper is generated by a basic block - a grid of black and white squares having three distinct columns and three to six rows, also distinct. There is a great deal of variation in appearance of these patterns, constructed as they are from simple rectangles of nine to eighteen black and white "tiles". Some have properties of optical illusions. Some seem to vibrate. Others, such as pattern 12, look very different when viewed from different angles, especially when observed obliquely. A consideration of the aesthetics of tilings is open to further

THE ELECTRONIC JOURNAL OF COMBINATORICS 15 (2008), \#R1 
study.

The requirement that a basic block have at least one black and one white square in each row and column comes from the necessity in weaving that each warp thread be interlaced with weft threads (and vice versa) as part of the fabric. We showed that this requirement is enough to ensure that a 2- or 3-harness fabric design hangs together.

Loosening the restriction that a generating block have distinct rows and columns expands the design possibilities. We can create interesting patterns, for instance, by incorporating weaving symmetries into a draft, as suggested by the representations of design families in Figures 8 through 14. Weavers often describe fabric design possibilities as "infinite" and they are of course correct if we do not restrict the number of rows or columns in the generating block. There are, however, many recurring themes in drafts of fabrics (and other ornamentation) that can be defined in terms of rectangular grids of finite dimension. This study is a first step of an inquiry into the number of weaving pattern patterns possible on a given number of harnesses, or the number of tilings that can be created from blocks with a given number of distinct columns.

The author has woven all 134 patterns in the 30 design families described in this report. Visual similarity of the cloth with the draft depends on factors such as the yarn used and the sett or spacing of the warp threads. Weavers know that not all drafts lead to satisfactory cloth. A woven sample may not resemble its draft in appearance because of physical properties of the yarn. The drafted fabric structure may lead to overlapping of adjacent weft threads and/or various distortions caused by differences in tension and interlacement. Some of these deviations from draft are expected and exploited by weavers to interesting effect [2]. Many of these phenomena are outside the realm of tiling and await mathematical attention.

Note: All figures in this article were created in Fiberworks [16] and edited using Windows Paint and Adobe Illustrator CS2.

Acknowledgements: Thanks to the reviewers, whose careful reading and detailed suggestions greatly improved the manuscript. Thanks also to Alina Stancu for helpful discussions and to Daniel Klain for sharing his enthusiasm for geometry.

\section{References}

[1] Anni Albers, On Weaving, Dover Publications, Mineola, New York, 2003; originally published by Wesleyan University Press, Middletown, Connecticut, 1965.

THE ELECTRONiC JOURNAL OF COMBINATORICS 15 (2008), \#R1 
[2] Sharon Alderman, Mastering Weave Structures, Interweave Press, Loveland, Colorado, 2004.

[3] Richard A. Brualdi and Herbert J. Ryser, Combinatorial Matrix Theory, Cambridge University Press, Cambridge, 1991.

[4] C.R.J. Clapham, When a fabric hangs together, Bulletin of the London Mathematical Society, Volume 12, 1980, 161-164.

[5] Cathy Delaney, When a fabric hangs together, Ars Combinatoria, Volume 21-A, 1986, 71-79.

[6] T.C. Enns, An efficient algorithm determining when a fabric hangs together, Geometriae Dedicata, Volume 15, 1984, 259-260.

[7] Branko Grünbaum and Geoffrey C. Shephard, Satins and twills: An introduction to the geometry of fabrics, Mathematics Magazine, Volume 53, 1980, 139-161.

[8] Branko Grünbaum and Geoffrey C. Shephard, A catalogue of isonemal fabrics, Annals of the New York Academy of Sciences, Volume 440, 1985, 279-298.

[9] Branko Grünbaum and Geoffrey C. Shephard, An extension to the catalogue of isonemal fabrics, Discrete Mathematics, Volume 60, 1986, 155-192.

[10] Branko Grünbaum and Geoffrey C. Shephard, Isonemal fabrics, American Mathematical Monthly, Volume 95, 1988, 5-30.

[11] Janet A. Hoskins, Factoring binary matrices; a weaver's approach, Lecture Notes in Mathematics, Volume 952, 1982, 300-326.

[12] Janet A. Hoskins, Cheryl E. Praeger and Anne Penfold Street, Twills with bounded float length, Bulletin of the Australian Mathematical Society, Volume 28, 1983, 255-281.

[13] Janet A. Hoskins, R. G. Stanton and Anne Penfold Street, Enumerating the compound twillins, Congressus Numerantium, Volume 38, 1983, 3-22.

[14] W.D. Hoskins and R.S.D. Thomas, Conditions for isonemal arrays on a cartesian grid, Linear Algebra and Its Applications, Volume 57, 1984, 87-103.

[15] Solomon W. Golomb, Polyominoes; Puzzles, Patterns, Problems, and Packings; revised and expanded second edition, Princeton University Press, Princeton, New Jersey, 1994.

[16] Bob Keates and Ingrid Boesel, Fiberworks PCW, Guelph, Ontario, 2005.

[17] C.L. Liu, Introduction to Combinatorial Mathematics, McGraw-Hill, New York, 1968.

[18] Janice Lourie, Loom-constrained designs: an algebraic solution, Proceedings of ACM National Conference, 1969, 185-192. 
[19] George E. Martin, Polyominoes; A Guide to Problems in Tiling, The Mathematical Association of America, 1991.

[20] Marks R. Nester, Sequential arrays, Utilitas Mathematics, Volume 51, 1997, $97-117$.

[21] Erica de Ruiter, Weaving on 3 shafts, Erica de Ruiter, Nijmegen, The Netherlands, 2002.

[22] J.E.A. Steggall, On the numbers of patterns which can be derived from certain elements, Messenger of Mathematics, 1908, 56-61.

[23] Anne Penfold Street and Deborah J. Street, Combinatorics of Experimental Design, Oxford University Press, Oxford, 1987.

[24] Marjie Thompson, Kathleen L. Grant and Alan G. Keyser, Forgotten Pennsylvania Textiles of the 18th and 19th Centuries, The Linen Press, Cumberland, Maine, 2005.

[25] John Tovey, Weaves and Pattern Drafting, Batsford, London, 1969.

[26] J.H. van Lint \& R.M. Wilson, A Course in Combinatorics, Second Edition, Cambridge University Press, Cambridge, 2001.

[27] Hermann Weyl, Symmetry, Princeton University Press, Princeton, 1952.

Department of Mathematical Sciences, University of Massachusetts Lowell, Lowell, MA 01854 USA; Shelley_Rasmussen@uml.edu 